\title{
Los tocados mayas en el discurso político. Un estudio de caso de los tocados de serpiente acuática durante el Clásico Tardío
}

\section{Maya Headdreses in Political Speech. A Case of Study of Waterlily Serpent Headresses during the Late Classic}

\author{
Ricardo Gómez Palacios \\ Posgrado en Estudios Mesoamericanos, \\ Universidad Nacional Autónoma de México, México
}

\begin{abstract}
Resumen: Este trabajo se centra en el estudio de los tocados mayas durante el periodo Clásico Tardío (650-900 d.C.) como objetos de identidad social. Para ello se llevó a cabo un estudio de caso de los tocados de serpiente acuática, los cuales fueron elementos visuales utilizados por una red de aliados encabezada por la dinastía Kanu'l durante el siglo vil. En este trabajo planteo que hay tres tipos de tocados de serpiente acuática que estuvieron relacionados con la dinastía Kanu'l, así como uno más que se vinculó con el señorío de Dos Pilas. A finales del Clásico Tardío los tocados de serpiente acuática se convirtieron en adaptaciones locales en diversos señoríos mayas que buscaban legitimar su posición en lo alto del orden sociopolítico maya.
\end{abstract}

Palabras clave: Tocados mayas, dinastía Kanu’l, serpiente acuática, iconografía, red política.

ABSTRACT: This paper focuses on the study of Maya headdresses as indicators of social identity during the Late Classic period. The study is centered in the Water Lily Serpent headdress, which were used by the Kanu'l dynasty and its allies during the $7^{\text {th }}$ century. I propose that three types of this motif were associated with the Kanu'l dynasty, while a fourth was related to the rulers of Dos Pilas. However, at the end of the Late Classic period the Water Lily Serpent headdresses were locally adapted in some other Maya dynasties with the intention of legitimize their position at the top of the regional sociopolitical hierarchy. 
KEYworDs: Maya headdresses, Kanu'l dynasty, Water Lily Serpent, iconography, political network.

ReCEPCIÓN: 19 de marzo de 2019.

ACEPTACIÓN: 14 de junio de 2019.

DOI: https://doi.org/10.19130/iifl.ecm.55.2020.0006

\section{Introducción}

En este artículo se revisa la importancia de los tocados mayas dentro de un discurso político que abarcaba las identidades sociales de quienes los portaban. Existen antecedentes de este tema que nos hablan del significado social y político de la vestimenta durante el periodo Clásico (Taylor, 1984; Le Fort, 2002; García y Vázquez, 2011; Tremain, 2017). Se pretende aquí problematizar este mecanismo general a través de un estudio de caso, como son los tocados de serpiente acuática durante el Clásico Tardío (650-900 d.C.), y se busca abordar la función de los tocados como elementos para identificar a sus portadores dentro de un grupo social, partiendo de la hipótesis de que pudieron ser marcadores de identidades políticas dentro de la sociedad maya. Para ello se realizó una revisión de monumentos mayas de este periodo, donde aparecen personajes portando tocados de la serpiente acuática. Una vez conformado el corpus, se analizó cada uno de ellos para obtener información que, desde una perspectiva comparativa, indicara patrones con respecto a la distribución espacial y temporal del tocado de la serpiente acuática, así como el género y rango social de quienes lo portaron. Con base en ello, el trabajo comprende tres partes. La primera nos habla de los tocados en general dentro del área maya, su simbolismo y significado; la segunda discute la información espacial y temporal de los tocados de serpiente acuática, y la tercera interpreta los patrones detectados con respecto al paisaje sociopolítico en las tierras bajas mayas.

\section{Los tocados mayas}

El tocado es el conjunto de elementos utilizado por un individuo sobre la cabeza, incluye plumas, diademas, mascarones u otros objetos de valor simbólico y estético (Morselli, 2004; Gómez, 2015). La función de estas prendas en el mundo prehispánico radicó en otorgar identidad y acentuar la posición jerárquica de quien lo portaba, otorgándole un estatus diferente al momento en el que era colocado (López Austin, 1973: 116; Ramírez y Arellano, 2001: 69; Grube, 2001: 72-73; Gómez, 2015: 44). Ejemplos de ello los tenemos en el Palacio de Tetitla, Teotihuacán, donde existe registro de la práctica de cierto ritual que culminaba con la colocación del tocado de la Deidad Ave Principal, legitimando así el mandato del portador 
mediante el cambio de estatus que adquiría al vestirlo (Helmke y Nielsen, 2015: 36). De igual forma, las composiciones de los tocados provenientes del Altiplano Central representaron animales como aves y jaguares, así como el famoso tocado de borlas que aparecerá en Teotihuacán y Tikal durante el Clásico Temprano, dato que ha ayudado a entender la conexión entre teotihuacanos y tikaleños (Paulinyi, 2001: 9).

En el caso maya, podemos apreciar en la Estela 22 de Naranjo al mandatario K'ahk' Tiliw Chan Chaahk con un tocado que lleva inscrito su nombre personal (Grube, 2001: 75). Si bien Stephen Houston y David Stuart (1998: 79) consideran que el gobernante está personificando al dios Chaahk, Nikolai Grube (2001: 74-76) propone que se trata del nombre personal del mandatario. Sin embargo, ambas propuestas se complementan, pues al adquirir el nombre K'ahk' Tiliw Chan Chaahk (literalmente "Chaahk que enciende el cielo con fuego"), el gobernante asume una relación identitaria con Chaahk y el tocado refleja esta fusión entre hombre y dios (Colas, 2004: XV). Dicho caso deja ver que el simbolismo de los tocados era muy complejo y podía expresar varios mensajes sobrepuestos.

Gracias a los trabajos arqueológicos se han encontrado tocados dentro de algunos recintos funerarios de mandatarios mayas. Este hecho es importante, pues nos habla de que los tocados fueron objetos materiales utilizados en la vida ritual y no se trata solamente de representaciones gráficas. Uno de estos ejemplares fue hallado en la tumba de Yihch'aak K'ahk', gobernante de la dinastía Kanu'l, en la Estructura II de Calakmul (Carrasco et al., 1999; Cordeiro, 2008; Carrasco, 2011). ${ }^{1}$ En el entierro se encontraron también restos de una máscara funeraria en la que se menciona que el portador personifica al dios Yax Chit Juun Witz' [Naah Kan], deidad que aparece como mascarón central en el tocado del fallecido, un dato que señala la armonía entre el ser personificado y el tocado portado. ${ }^{2}$

La personificación fue una de las prácticas recurrentes durante el Clásico Tardío para legitimar la posición sagrada del gobernante frente a la población (Stone, 1986: 194). El soberano realizaba un ritual que lo colocaba en lo alto de la estructura sociopolítica del mundo maya mediante la obtención de una carga divina (Nehammer, Thun y Helmke, 2009: 187). Una entidad sobrenatural poseía a los individuos, que seguían una serie de pasos para lograrlo, y que nunca perdían su esencia pues "a pesar de que los figurantes estaban poseídos por los dioses y mantenían una parte de su espíritu externado, asumían la responsabilidad moral de sus acciones" (Velásquez, 2010: 221). Los tocados eran parte medular de los rituales que buscaban establecer un vínculo con lo sagrado (Velásquez, 2010: 215),

${ }^{1}$ Otro ejemplo lo tenemos en la Estructura L5-1 de la plaza principal de Dos Pilas, donde fue colocada la tumba de Kokaaj K'awiil, segundo gobernante de la ciudad (Demarest et al., 1992).

${ }^{2}$ De igual manera ocurre en el Tablero del Templo XIX de Palenque, donde se menciona que fueron personificados los dioses GI e Itzam Naah Yax Kokaaj Muut, los cuales portan tocados que llevan la cabeza del dios, mostrando así la relación entre la escritura y la iconografía (Boot, 2008: 22; Nehammer, Thun y Helmke, 2009: 191; Velásquez, 2010: 207). Lo mismo ocurre en el Dintel 5 del Sitio R, donde el tocado le da el nombre al ritual practicado por los personajes (Looper, 2004: 2). 
así como relacionar a los mandatarios con su pueblo y, a la vez, marcar y reforzar la jerarquía existente entre los distintos grupos sociales (Durkheim, 1964: 375). Es por ello que dichas prácticas eran ejecutadas en lugares de encuentro social con el objetivo de ser presenciadas por un público determinado (García y Valencia, 2007: 34-35). Los rituales de personificación son señalados en algunos casos con elementos iconográficos, como sería el glifo T533 (AJAW) que aparece en lo alto de algunos tocados (Velásquez, 2010: 217). Con base en esto, se sugiere que la colocación del tocado era la parte culminar del ritual de personificación, cuando el agente humano seguía una serie de pasos que le permitían portarlo para señalar que estaba siendo poseído por el dios invocado.

Los dioses personificados pueden categorizarse en dos tipos, los de culto general y los patronos, que fungían como protectores de un linaje y se veneraban con alimentos y dedicación de edificios (Baron, 2016: 76, 85). La poca información que se conserva del periodo Clásico no permite explicar por qué había una jerarquía entre los dioses patronos (García Capistrán, 2014: 75, 79). No obstante, se sabe que se utilizaron para pactar alianzas y legitimar a otros gobernantes, pues en el Panel 1 de Cancuén y en la Estela 35 de Piedras Negras es mencionado Ch'e'n II de Calakmul acompañado del dios patrono de los Kanu'l, Yax Ha'al Chaahk, con el fin de legitimar a los recién entronizados (García Barrios, 2014: 59; Baron, 2016: 168-169). La imagen de los dioses patronos también fue utilizada para legitimar la posición del gobernante frente a otros grupos, tal es el caso del Dintel 6 de Tikal, donde se representa a Jasaw Chan K'awiil portando un traje del dios patrono K'in Bahlam, con el objetivo de mostrarse como un gobernante apoyado por el dios patrono del linaje (Le Fort, 2002: 34).

En otros casos, como el del traje de red, se ha estudiado que fue una prenda utilizada por mujeres relacionadas con la dinastía Kanu'l (Reese-Taylor et al., 2009; García y Vázquez, 2011). Si bien los primeros ejemplares del Clásico Temprano fueron utilizados como un elemento pan maya, es durante el Clásico Tardío cuando se tiene suficiente información para pensar que los Kanu'l, en su afán expansionista, utilizaron el traje de red como un código de vestimenta entre sus aliados con el fin de generar una cohesión bajo el símbolo de su dios patrono impreso en el atuendo (García y Vázquez, 2011). El traje de red parece vincularse al mito del dios del maíz (García y Vázquez, 2011: 81-82; Helmke y Kupprat, 2016: 47), pero también representaba a otras deidades acuáticas ${ }^{3}$ como GI, la diosa de la luna (Reese-Taylor et al., 2009) y Yax Chit Juun Witz' Naah Kan, dios patrono de la dinastía Kanu'l (Baron, 2016: 173). Vemos en la Estela 34 de El Perú y en el Panel 6 de la Corona a mujeres con trajes de red relacionadas con la dinastía Kanu'l cuyo objetivo era pactar alianzas con los señoríos locales mediante el matrimonio. Con base en esto, se puede decir que las prendas con motivos de dioses patronos, como trajes - e incluso tocados, de los que se hablará más adelante-, eran utilizados tanto dentro como fuera de las ciuda-

\footnotetext{
${ }^{3}$ Felix Kupprat, comunicación personal, 2018.
} 
des con el fin de crear alianzas y legitimar señoríos locales (Le Fort, 2002: 34; Tremain, 2017: 112).

La vestimenta fungió como un marcador social que podía englobar a grupos dentro de un mismo núcleo. Alexander Parmington (2003: 49-52), a través de su estudio de los sajal mayas, propone que había un código de vestimenta exclusivo de este grupo que los diferenciaba del resto de la corte maya. ${ }^{4}$ Con base en tales datos, Cara Tremain (2017: 140) propone que la vestimenta maya tuvo una función social, que se dividía en tres tipos: uniformes de grupos sociales que son utilizados por grandes grupos, como los sajal; de rol individual, que sólo era ocupado por una persona, como el k'uhul ajaw o el kalo'mte', y como grupo social, utilizados para unificar grupos de diversas ciudades o señoríos mayas sin importar títulos. ${ }^{5}$ Con base en ello, se puede decir que los tocados en el área maya fueron importantes símbolos cuyo significado englobaba la vida política y religiosa de las sociedades. Su presencia no sólo tenía fines estéticos sino que respondía a la creación de un canon visual que abarcaba toda la parafernalia del individuo que lo portaba. ${ }^{6}$

\section{Contexto sociopolítico maya del Clásico y estrategias de integración}

Algunas investigaciones sobre la configuración sociopolítica de los sitios clásicos en el área maya se han enfocado en el entendimiento de las entidades políticas regionales llamadas súper estados o estados hegemónicos. A partir del trabajo de Simon Martin y Nikolai Grube (1994, 2008: 20-21) se ha prestado mayor atención a la conformación de los dos estados hegemónicos más extensos en el Clásico Tardío, con centros en los sitios de Tikal y Calakmul, los cuales establecieron alianzas, vasallajes ${ }^{7}$ y pactos con otros sitios en el área maya (Martin y Grube, 2008: 16-18).

\footnotetext{
${ }^{4}$ Algo similar ocurre en la cultura mexica con los guerreros jaguar y águila, los cuales utilizaban determinados atuendos para marcar su rango militar dentro de la sociedad.

${ }^{5}$ Habría que agregar uno más que es el de los prisioneros, los cuales eran despojados de sus prendas con el objetivo de desprestigiar al cautivo. Un ejemplo de ello ocurre con el k'uhul ajaw de Palenque, K’an Joy Chitam, quien, al ser capturado por Toniná, es representado sin su parafernalia y sin el adjetivo K'ihnich (caliente) de su nombre (Tremain, 2017: 116), dejándonos ver que los cautivos perdían ciertos atributos de su persona al caer en esa condición.

${ }^{6}$ En Yaxchilán, por ejemplo, el tocado, la vestimenta y los objetos que lleva Yaxuun B’ahlam IV responden a un patrón de acompañamiento. Dicho patrón se puede ver en los Dinteles 1, 53 y 54 donde se representa el baile del K'awiil. En las tres escenas el protagonista utiliza un mismo tocado y una misma vestimenta; mientras que en otros dinteles ( 2 y 5 ) se ilustra al mismo personaje practicando el ritual conocido como flap-staff birds, en donde el tocado y la vestimenta es diferente a las primeras tres escenas (Gómez, 2015: 157-162). Lo mismo ocurre en monumentos de Machaquilá y Dos Pilas, de los cuales hablaré más adelante.

${ }^{7}$ En los textos jeroglíficos aparecen expresiones como yajaw, 'su señor', y ukabjiily, 'bajo los auspicios de', indicando que algunos sitios fueron subordinados de Calakmul o Tikal (Martin y Grube, 1994, 2008).
} 
Dos ejemplos de las alianzas que se establecieron en Calakmul se han registrado en La Corona y El Perú, a donde fueron enviadas mujeres para contraer nupcias con los mandatarios locales (Martin, 2008; Canuto y Barrientos, 2010; 2013: 1; García Capistrán, 2012: 47; Acuña, 2014: 63; Vázquez, 2015, 2017). Además, en la Escalinata Jeroglífica 4 de Dos Pilas se mencionó que Bajlaj Chan K'awiil, fundador de la casa gobernante local, fue el yajaw de Ch'e'n II de Calakmul (Houston y Mathews, 1985: 14; García Capistrán, 2012: 53). Estos tres centros manifestaron en sus inscripciones una clara relación con los señores Kanu'l, dinastía asentada en el sitio de Calakmul en el Clásico Tardío. No obstante, en Palenque y Yaxchilán es difícil afirmar que los Kanu'l tuvieron una presencia significativa en la política local, pues en el primero se menciona que Janaab Pakal gobernó la ciudad tras un ataque organizado por los gobernantes Kanu'l (Grube, 1995: 5; Martin y Grube, 2008: 156-161; Bernal, 2011: 36); mientras que en Yaxchilán, se indica que Yaxuun Bahlam IV fue hijo de una Ix Kanu'l (Mathews, 1997: 195). Sin embargo, los textos no ofrecen más información en torno a la posible presencia de los gobernantes de Calakmul en las mencionadas dinastías.

La integración a la red política desarrollada por los Kanu'l durante el siglo VII, se logró mediante matrimonios y pactos, intercambio de regalos, banquetes y ritualidad compartida, en donde los miembros de las dinastías participantes utilizaban elementos visuales que los identificaban como miembros de un grupo (Vázquez, 2015, 2016, 2017). Tal es el caso de las estelas pareadas que representan a parejas gobernantes, así como paneles del juego de pelota que aparecen en los espacios construidos por los Kanu'l y sus aliados (Helmke et al., 2015: 1, 8, 12 y 19; Vázquez, 2015, 2016, 2017). Otros ejemplos de ritualidad compartida ${ }^{8}$ fue el envío de gobernantes de otros sitios mayas a la ciudad de Calakmul para ser entronizados y legitimar sus mandatos, ${ }^{9}$ el culto a ciertos dioses y la creación de protocolos visuales ya mencionados.

Tras la muerte de Yihch'aak K'ahk' en 698 d.C., la red política de Kanu'l fue decayendo de manera gradual durante el siglo vilI (Martin y Grube, 2008: 111). Esto llevó a que las estrategias de integración disminuyeran poco a poco, pues ya para el siglo ix es difícil relacionar las prácticas mencionadas arriba con los Kanu'l. No obstante, aún para dicha temporalidad vemos ciudades ubicadas en el Usumacinta, Petexbatún y Pasión llevando a cabo estas prácticas, las cuales pudie-

\footnotetext{
${ }^{8}$ El ritual de la toma del K’awiil fue de gran importancia para la dinastía Kanu’l, el hallazgo del Panel 4 de Xunantunich ha llevado a especialistas a proponer que hay una relación entre este ritual y la dinastía Kanu'l, pues los eventos de entronización que son presenciados por los miembros de la mencionada dinastía, por lo regular incluyen el término cham K'awiil, 'tomar el K'awiil'. No obstante, nuestro conocimiento acerca de tal relación dista de ser profundo como para afirmar que es un ritual propio de los Kanu'l, pues la vemos como una práctica común del área maya en épocas tempranas que pudo ser apropiada por un grupo de élite (Helmke y Awe, 2016: 14-17).

${ }^{9}$ Un ejemplo se encuentra contenido en el Panel 2 de La Corona, donde se menciona que el heredero al trono, K'ihnich Ahkan? Yook fue a Calakmul para ser entronizado y vincular así su gobierno con el de una dinastía más poderosa como lo fueron los Kanu'l (Stuart et al., 2013: 439).
} 
ron ser adaptaciones post-Kanu'l a fin de otorgarle legitimidad a sus gobiernos, de lo cual se hablará más adelante.

\section{Los tocados de serpiente acuática: características iconográficas}

La serpiente acuática aparece en diversas escenas palaciegas mayas a lo largo de toda la época prehispánica, y su presencia señala que la escena representada se llevó a cabo en un lugar acuático (Ishihara, Taube y Awe, 2006; Acuña, 2014: 63). La deidad que encarna la serpiente acuática es conocida como Yax Chit Juun Witz' Naah Kan, 'primera serpiente de agua' o 'gran serpiente de la cascada' (Stuart, 2007; Lacadena, 2011: 231; Zamora, s.f.: 75-94). ${ }^{10}$ Gracias a los avances epigráficos se ha leído en la Estela 54 de Calakmul que Yax Chit Juun Witz' Naah Kan fue un dios patrono de la dinastía Kanu'l, para el cual se ha reconstruido un culto estatal en Calakmul (Baron, 2016: 173; Vázquez y Kupprat, 2018: 90-91). Ligado a esto, en el Dintel 28 de Yaxchilán se menciona a una Ix Kanu’l Ajaw, madre de Yaxuun Bahlam IV, que integra el téonimo facultativo de Juun [Witz'] Naah Kan en su fase nominal. De igual forma, el Monumento 2 de Calakmul menciona a un señor Kanu'l que integra el teónimo [...] Naah Kan en su nombre (Vázquez y Kupprat, 2018: 90), así como en la máscara funeraria de Yihch'aak K’ahk' ya mencionada. Estos datos, provenientes del Clásico Tardío, indican una relación entre la deidad acuática y la dinastía Kanu'l durante tal periodo, es por ello que el presente escrito parte de una relación existente entre la mencionada dinastía y los tocados de serpiente acuática.

Ahora bien, los tocados de serpiente acuática aparecen en la iconografía maya desde el Preclásico; hay ejemplares de éstos en el Mural Oeste de San Bartolo, la Estela 5 de Izapa y la Vasija K 4562. Mientras que para el Clásico Temprano se pueden ver en la Estela 1 de Caracol y la Estela 5 de Naranjo. No obstante, es en el Clásico Tardío cuando tenemos una gran cantidad de representaciones. Los rasgos diagnósticos de los tocados de serpiente acuática se ven notoriamente en la vasija K1162 (Figura 1A) (Kerr, 2008: K1162), y son una cabeza de ofidio con labio superior hacia abajo (W), ${ }^{11}$ que porta un tocado con un pez (Z), el cual mor-

\footnotetext{
${ }^{10}$ La traducción de Yax Chit es problemática, pues si bien yax, puede referirse a 'primero' o 'verde', no hay una traducción aceptada para chit. Erik Velásquez García (2006: 135) propone que la frase yax chit pudo ser utilizada para referirse a ofidios que aparecen en la iconografía maya. Otra propuesta indica que es una expresión genérica que resalta una función de los dioses patronos (personales) o compañeros (Prager, 2013: 518-525, citado en Kupprat, 2015: 291).

${ }^{11}$ Es importante señalar que el labio superior de la serpiente, tanto en su forma glífica como iconográfica, se representó de dos maneras, con el labio girando hacia arriba y girando hacia abajo. Sin embargo, se desconoce el significado de cada variante, pues puede tratarse de dos tipos de serpientes cuyo significado aún desconocemos. Ahora bien, debemos tener cuidado y no confundir a la serpiente acuática de labio superior girado hacia abajo con la Deidad Ave Principal que aparece frecuentemente en escenas del Clásico, pues aunque ambos seres comparten algunas características iconográficas como el labio superior y la mirada con estrabismo, el Ave Principal siempre lleva con-
} 
disquea una flor de nenúfar $(\mathrm{Y})$ atada a una concha $(\mathrm{X})$. En otras representaciones del Preclásico y el Clásico Temprano se puede apreciar una serpiente acuática con ligeras variantes. No obstante, este ejemplar proveniente de un diente de un felino (Figura 1B) ilustra a una serpiente de labio superior alargado hacia arriba (U), con un tocado que en lo alto lleva una voluta (V) posada sobre una flor de nenúfar $(\mathrm{Y})$ atada a una concha $(\mathrm{X})$. Ambas representaciones de cabezas de serpiente acuática nos dejan ver similitudes entre ellas que son consideradas como elementos diagnósticos y se señalan en la Tabla 1.

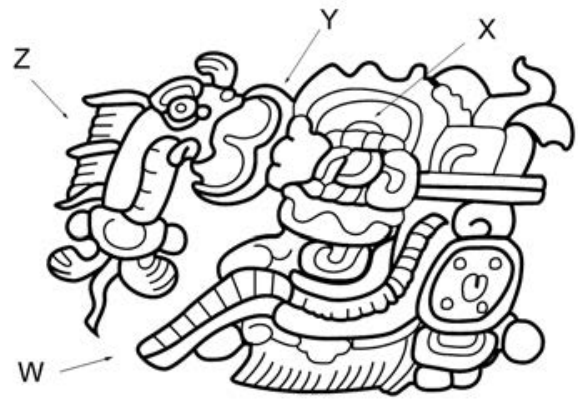

A

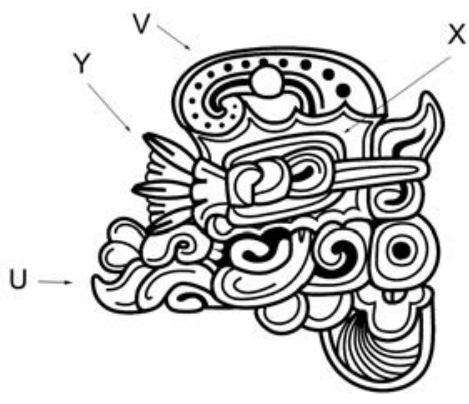

B

Figura 1. Tocados de serpiente acuática con elemento diagnóstico.

A: vasija Kerr 1162, basado en dibujo de Linda Schele y Mary Ellen Miller (1986).

B: diente de felino, basado en dibujo de José Franco (Houston, 2017)

(Dibujos de Ricardo Gómez Palacios).

\begin{tabular}{|l|l|l|l|}
\hline \multicolumn{2}{|c|}{ Figura 1A } & \multicolumn{2}{c|}{ Figura 1B } \\
\hline Cabeza de ofidio labio hacia abajo & W & Cabeza de ofidio labio hacia arriba & U \\
\hline Pez & Z & Volutas & V \\
\hline Nenúfar & Y & Nenúfar & Y \\
\hline Concha $^{12}$ & X & Concha & X \\
\hline
\end{tabular}

Tabla 1. Rasgos diagnósticos de la serpiente acuática.

sigo adornos nasales y un medallón en la frente como rasgos diagnósticos; en el tablero del Templo XIX de Palenque se representa a esta deidad en forma de tocado (Kerr, 2008: K7821, K3863).

${ }^{12}$ He señalado al elemento " $X$ " como una concha, pues iconográficamente hay un gran parecido con las conchas marinas. Sin embargo, hay otros ejemplares de tocados de serpiente acuática donde la concha es sustituida por un caparazón de tortuga (Zamora, s/f: 20). Algunos autores lo consideran como un caparazón de tortuga que algunas veces es sustituido por una concha (Schele y Miller, 1986: 46; Ishihara, Taube y Awe, 2006: 212). La razón de ello no ha sido explicada, pero intuyo que ambos elementos (la concha y el caparazón) están relacionados con el mundo acuático, por lo que no hay como tal un cambio sustancial en el significado, pues ambos tienen un mismo motivo. Cabe señalar que en la variante 3 aparece otro tipo de concha, dadas las diferencias iconográficas entre ellas, he decidido catalogar a la concha de las variantes 1 y 2 con la letra "X"; mientras que a la de la variante 3 con la letra "S", buscando así una mayor precisión en la clasificación. 
Con base en los elementos diagnósticos señalados en la tabla, se han identificado tres variantes de tocados de serpiente acuática, señalados en la Tabla 2. Estos tres tocados los encontramos distribuidos a lo largo del territorio maya durante el Clásico Tardío y fueron utilizados por diversos miembros de la élite.

\begin{tabular}{|l|l|l|l|l|l|}
\hline Variante $1^{13}$ Figura 2 A-C & \multicolumn{4}{l|}{ Variante $2^{14}$ Figura 3 A-C } & \multicolumn{2}{l|}{ Variante $4^{15}$ Figura A-C } \\
\hline Ofidio labio hacia abajo & W & Pez & Z & Ofidio labio hacia arriba & U \\
\hline Pez & Z & Nenúfar & Y & Concha & S \\
\cline { 1 - 4 } Nenúfar & Y & Concha & X & Ofidio nariz cuadrada & T \\
\cline { 1 - 2 } Concha & X & & Bandas cruzadas & R \\
\cline { 1 - 2 } & & Marca de brillo ${ }^{16}$ & Q \\
\hline
\end{tabular}

Tabla 2. Rasgos diagnósticos de las tres variantes de serpiente acuática.

\section{Distribución espacial y temporal de los tocados de serpiente acuática}

Los tocados de variante 1 (Figura $2 \mathrm{~A}-\mathrm{C}$ ) fueron utilizados por hombres y mujeres gobernantes, así como por algunos sajal a lo largo de las tierras bajas centrales mayas. Los tocados de variante 2 (Figura $3 \mathrm{~A}-\mathrm{C}$ ) se aprecian en gobernantes de

${ }^{13}$ Algunos ejemplares del Clásico Temprano aparecen a manera de glifo en las Estelas 63 (435 d.C.) (Stuart et al., 1989: Figura 1) y 24 de Copán (485 d.C.) (Morley, 1920: Figura 7). En ambas ilustraciones se aprecia una pequeña voluta sobre la cabeza que acompaña el conjunto, la cual desaparece en los ejemplos del Clásico Tardío y ha sido considerada como una voluta o rizo de agua o bien, una nube (Daniel Salazar Lama, 2019, comunicación personal).

${ }^{14}$ Esta variante está ausente de un mascarón. Es probable que se trate de un pars pro toto de la variante 1 . Sin embargo, he decidido presentarlos como una variante aparte para hacer un análisis más minucioso de los tocados.

15 Los tres elementos del tocado de variante $3(\mathrm{~S}, \mathrm{~T}$ y $\mathrm{R})$, son similares al tocado que porta el llamado monstruo cuadripartita (Robertson, 1974: 77) que aparece en monumentos de Copán como la Estelas H e I (Robertson, 1974: 92, Figuras 31-32), así como en el Templo de la Cruz de Palenque (Robertson, 1974: 77, Figura 1) y la Lápida funeraria de Pakal. En estos últimos, un axis mundi en forma de árbol emerge del tocado del monstruo, dejando ver elementos relacionados con la naturaleza (Stuart, 2005: 164). Los tocados de variante 3 y el monstruo cuadripartita que se representa en los monumentos mencionados, tienen ligeras diferencias iconográficas, la más notoria de ellas es que el monstruo lleva en la frente inscrito el glifo K'IN y una espina de raya sobre su cabeza en lugar de la serpiente de nariz cuadrada. Ambos seres (monstruo y serpiente acuática) están relacionados con la iconografía del dios GI y con las mujeres durante el Clásico Tardío. El tocado del monstruo cuadripartita y los de variante 3 aparecen representados constantemente con mujeres y el dios GI durante el Clásico Tardío, lo que ha sido interpretado como símbolos vinculados al mundo acuático (Stuart, 2005: 169-170). Algunos tocados de variante 3 pertenecientes al Clásico Temprano se encuentran en la Estela 2 de Tikal y en la Estela 5 de El Zapote, que ilustra a un personaje que además del tocado porta un traje de red.

${ }^{16}$ Un caso que causa conflicto es el de la Estela 24 de Xultún, donde se representa un tocado del monstruo cuadripartita con una mujer que lleva un traje de red; faltaría analizar con más detalle el significado del monstruo en los tocados y la diferencia política con la variante 3 . 
ciudades y en aquellos que ostentaron el título de sajal. En la mayoría de las escenas se muestra a los portadores practicando el juego de pelota y, en algunas otras, con glifos calendáricos sobre sus manos. Para el caso de los tocados de variante 3 (Figuras $4 \mathrm{~A}-\mathrm{C}$ ), son las mujeres las únicas que se representaron utilizándolos, quienes además visten en su mayoría un traje de red, que fungiría como un protocolo visual de orientación política.
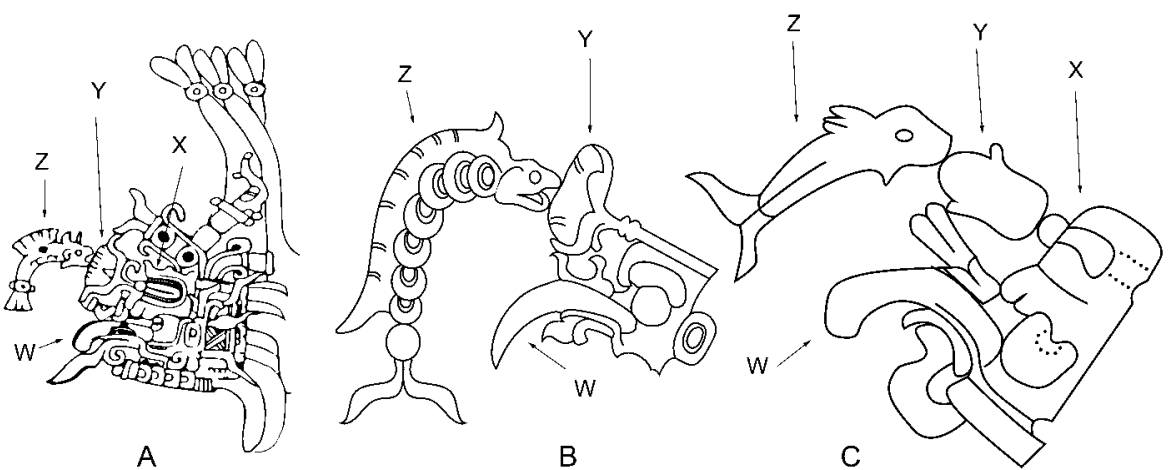

Figura 2. Tocados de serpiente acuática de variante 1. A: Estela 2 de Bonampak;

B: Estela 18 de Machaquilá y C: Dintel 6 de Yaxchilán. A, basado en dibujo de Peter Mathews (1978); B, basado en dibujo de Berthold Riese

(Chocón y Laporte, 2002); C, basado en dibujo de Ian Graham (1982)

(Dibujos de Ricardo Gómez Palacios).

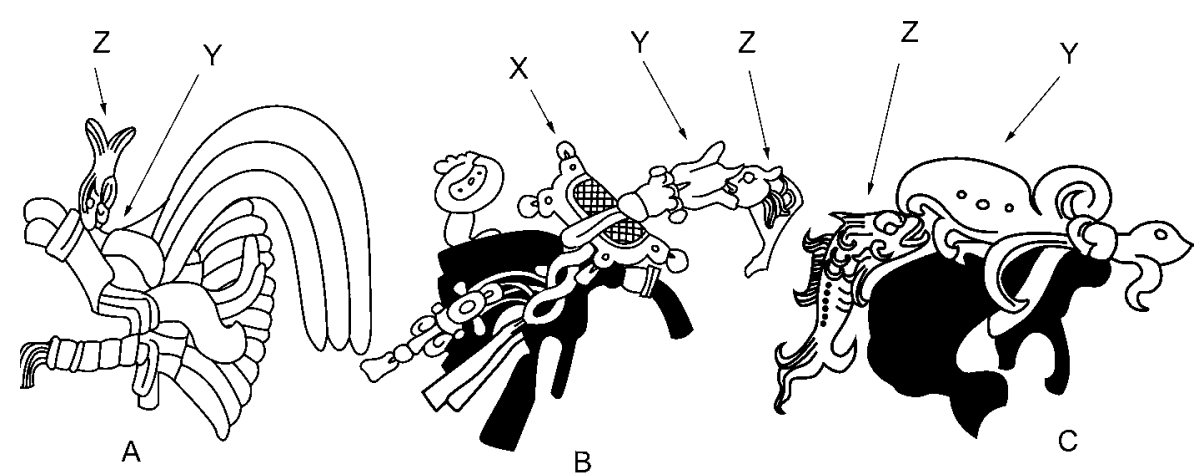

Figura 3. Tocados de serpiente acuática de variante 2. A: Dintel 2 de Laxtunich; B: Panel 1 de Pomoná y C: Tablero del Palacio de Palenque. A, basado en dibujo de Linda Schele (Jackson, 2013); B, basado en dibujo de David Stuart e Ian Graham (Stuart, 2004); C, basado en dibujo de Linda Schele (1979) (Dibujos de Ricardo Gómez Palacios). 


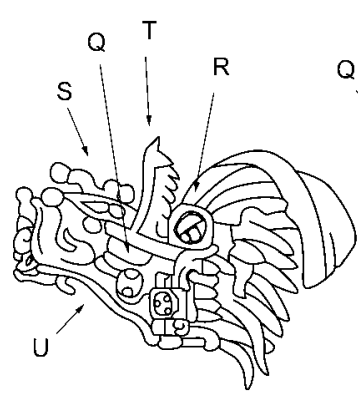

A

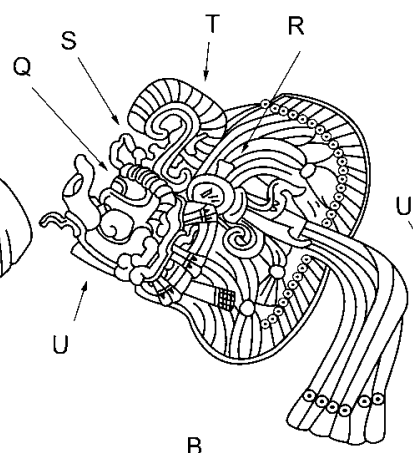

B

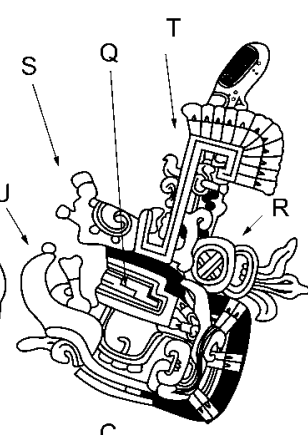

C

Figura 4. Tocados de serpiente acuática de variante 3. A: Vasija Schaffhausen; B: Estela 1 de El Chorro; C: Estela 34 de El Perú. A, basado en dibujo de Christian Prager (2004); B, basado en dibujo de Nikolai Grube (Mayer, 1989); C, basado en dibujo de John Montgomery (Wanyerka, 1996) (Dibujos de Ricardo Gómez Palacios).

Los primeros tocados de serpiente acuática representados durante el Clásico Tardío se hallan en la zona del Petén. La Estela 9 de Calakmul ilustra a un personaje llamado Ho' Hu'n? Sipiil? portando un tocado de variante 1, mientras que en la vasija de Schaffhausen aparece una mujer, cuyo nombre no ha sido descifrado, con un tocado de variante 3 durante el siglo viI (Figuras 5A, 5B y 6).

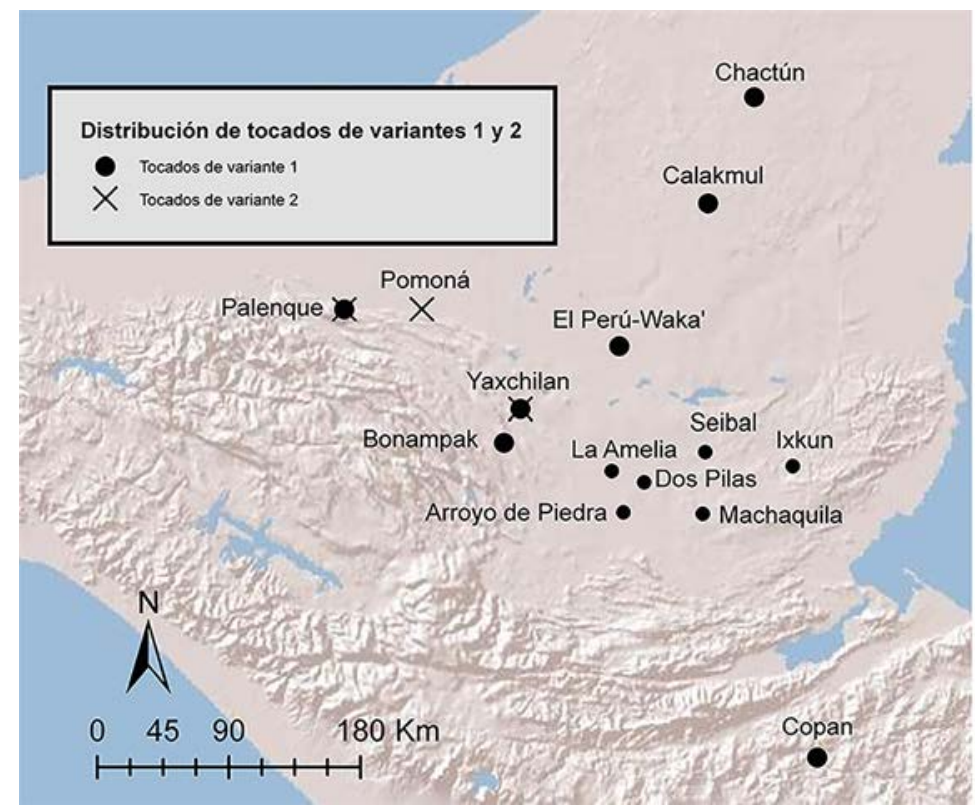

Figura 5. Mapas de distribución de los tocados de serpiente acuática. A: tocados de variantes 1 y 2 . 


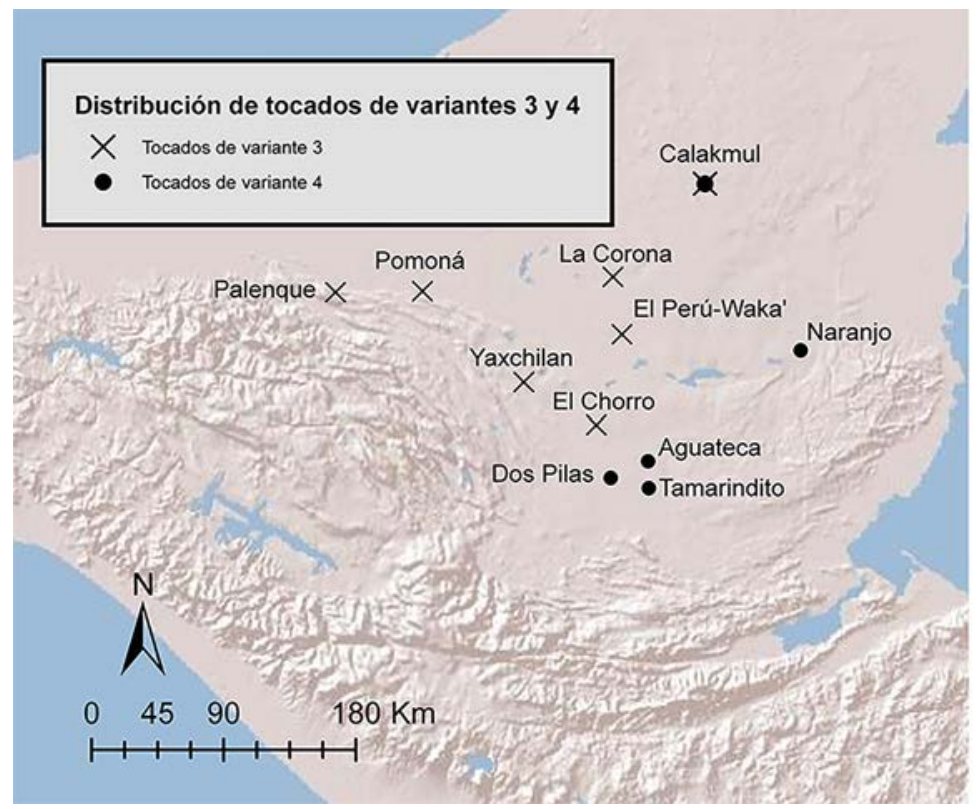

Figura 5. Mapas de distribución de los tocados de serpiente acuática. B: tocados de variantes 3 y 4 .
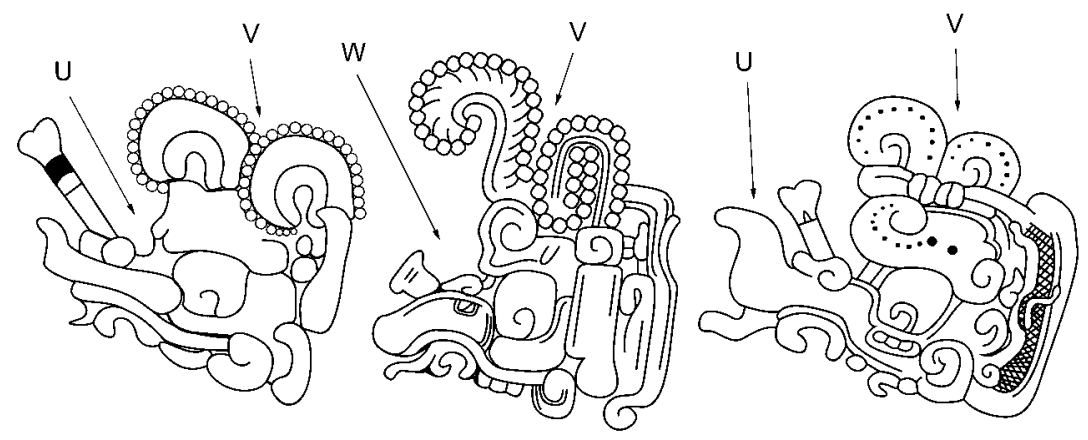

Figura 6. Tocados de serpiente acuática de variante 4. A: Estela 17 de Dos Pilas; B: Estela 13 de El Naranjo; C: Estela 3 de El Tamarindito. A, Basado en dibujo de Linda Schele (Vega, 2014); B, basado en dibujo de Ian Graham (Graham y Von Euw, 1975); C, basado en dibujo de Ian Graham (Gronemeyer, 2013)

(Dibujos de Ricardo Gómez Palacios).

En El Perú aparece el gobernante local, Bahlam II, portando un tocado de variante 1 en la Estela 33 con fecha 692 d.C. En su monumento par, la Estela 34, se observa a su esposa proveniente de Calakmul, Ix K'abel, quien porta un tocado, y además lo personifica, de Yax Chit Juun Witz' Naah Kan. En los paneles 3 (662 
d.C.) y 6 (731 d.C.) de La Corona se ilustraron a dos mujeres relacionadas con la dinastía Kanu'l portando tocados de variante 3. Es importante mencionar que en los sitios de La Corona y El Perú comenzaron a representarse personajes con tocados de serpiente acuática al establecerse la alianza con los Kanu'l.

En las tierras bajas centrales y el Usumacinta hay una presencia notoria de tocados de serpiente acuática. En Palenque se conservan tres monumentos elaborados después del mandato de Janaab Pakal que ilustran personajes con tales tocados. El primero de ellos es un portaincensario con el rostro de la sacerdotisa Ix Aj Pay K'ab que lleva un tocado de variante 1 . El segundo ejemplar, de variante 3, es llevado por la esposa de Janaab Pakal, Ix Tz'akbu Ajaw, en el Tablero del Templo XIV; mientras que el último es un tocado de variante 2, que aparece en el Tablero del Palacio utilizado por Janaab Pakal. En el Tablero del Templo XIV, Ix Tz'akbu Ajaw porta además un traje de red. Lo mismo ocurre en Pomoná, donde se aprecia a Ix K'an Balun con un tocado de serpiente acuática de variante 3 con traje de red en el Elemento 30 y el Panel de Dallas. Otros tocados de serpiente acuática de variante 2 aparecen en los Paneles 3 y 1 , de los cuales sólo se ha fechado el último para el 731 d.C.

En Yaxchilán hay tocados de serpiente acuática de las variantes 1, 2 y 3 que fueron representados durante el mandato de Yaxuun Bahlam IV (752-768 d.C.). Los tocados de variante 1 fueron utilizados por Yaxuun Bahlam y sus sajal, mientras que la variante 3 fue portada por Ix Uh Chan Lem?, quien, de acuerdo con la lectura de la Estela 10 de Yaxchilán, pertenecía al linaje de los Kanu'l (Mathews, 1997: 173). En el Dintel 2 de Laxtunich se representa a Kokaaj Bahlam IV, hijo de Yaxuun Bahlam IV, con un tocado de variante 2 representándose a sí mismo como la manifestación de Yax [Chit] Juun Witz' [Naah Kan]; mientras que en la Estela 2 de Bonampak, se aprecia a una mujer con un tocado de variante 1 personificando a Yax Chit Juun Witz' Naah Kan, misma que ha sido identificada como la hija de Yaxuun Bahlam IV. Otro miembro de la élite de Yaxchilán que utilizó estos tocados fue la señora Ix Chak Joloom, esposa de Yaxuun Bahlam IV, quien personifica a la misma deidad en el Dintel 1 de Yaxchilán. Este caso deja ver una cohesión familiar en la que la abuela, el padre (Yaxuun Bahlam IV), la madre y los hijos se atavían con tocados de serpiente acuática y personifican a Yax Chit Juun Witz' Naah Kan en diversos monumentos.

En las zonas del Petexbatún y el río de La Pasión, los tocados de serpiente acuática fueron utilizados entre los siglos vill y Ix. Una de las ciudades donde hubo una clara influencia de los Kanu'l es Dos Pilas, donde se aprecia a Kokaaj Bahlam con un tocado de variante 1 en la Estela 15. Otros sitios donde se representaron tocados de serpiente acuática fueron Machaquilá, Arroyo de Piedra, Ixkún, Ceibal y La Amelia. Por último, hay dos ejemplares más de tocados de la variante 1 que aparecen durante el siglo vilI. El primero de ellos es la Estela $\mathrm{N}$ de Copán, ubicado cerca del río Motagua, mientras que en la Estela 18 de Chactún se distingue a un personaje antropomorfo con el mencionado atributo. 


\section{Discusión}

La distribución espacial y temporal de las tres variantes de tocado de serpiente acuática estuvo centrada en tres zonas. La primera de ellas es el Petén durante el siglo vII, en donde la dinastía Kanu'l tenía una gran influencia política dentro de las tierras centrales bajo el mandato de Ch'e'n II (Martin y Grube, 2008: 109). Aquí los portadores de estos tocados mostraron un vínculo sanguíneo entre los Kanu'l y sus aliados representados en La Corona y El Perú.

La segunda zona identificada es la parte occidental de las tierras bajas, donde es complicado hablar de una influencia directa de los Kanu'l, pues en el caso de Palenque ninguno de los portadores personifica a Yax Chit Juun Witz' Naah Kan. No obstante, la presencia de Ix Tz'akbu Ajaw de Palenque e Ix K'an Balun de Pomoná con traje de red y tocado de variante 3 durante el siglo vI, fecha paralela al auge hegemónico de los Kanu'l (siglo vil) (Bernal, 2011: 61), abre la posibilidad que ambos sitios recibieron influencia política de ellos. Ahora bien, para Yaxchilán se tiene el registro de que durante el siglo vill la familia gobernante se representó personificando a Yax Chit Juun Witz' Naah Kan, fecha en la que el poderío de los Kanu'l estaba en decadencia, lo que me lleva a pensar en dos posibilidades. Por un lado, se infiere que hubo influencia de los Kanu'l en la política interna de Yaxchilán durante el mandato de Yaxuun Bahlam IV, misma que se vio reflejada en la práctica de rituales compartidos y en la madre del gobernante que se nombra a sí misma como Ix Kanu'l Ajaw. Por otro lado, puede que en realidad se tratara de un recurso retórico utilizado por Yaxuun Bahlam IV para legitimar su posición como gobernante de la ciudad mediante la práctica de ritos y el uso de títulos relacionados con los Kanu'l. En ambos casos, se refuerza la idea de que el tocado, así como la personificación de Yax Chiit Juun Witz' Nah Kan, estuvieron relacionados con la dinastía Kanu'l que, de alguna forma directa o indirecta, estaba involucrada en la política local del Usumacinta. Ligado a esto, el hecho de que la aparición del tocado en Yaxchilán coincida, más o menos, con la representación de Ix Uh Chan Lem?, señora de Kanu'l, refuerza la idea de una unidad política bajo el uso de este tipo de tocados en la zona. ${ }^{17}$ Lo mismo ocurre con Palenque y la entronización de Pakal tras la invasión de Calakmul en el siglo viı d.C.

La tercera zona donde aparecen tocados de serpiente acuática son las cuencas del Petexbatún y La Pasión; todos sus sitios, además de encontrarse muy cerca geográficamente hablando, representaron a gobernantes portando tocados de serpiente acuática en su variante 1 a finales del siglo vil y principios del siglo ix. Aquí es complicado hablar de una presencia Kanu'l dentro de la política interna, ${ }^{18}$ por lo que en realidad podría tratarse de una fase post-Kanu'l con el fin de legitimar los gobiernos locales a través de relacionar su señorío con otro

\footnotetext{
${ }^{17}$ Los únicos dos ejemplares que provienen de Laxtunich y Bonampak son miembros de la familia de Yaxchilán.

${ }^{18}$ Salvo el caso de Dos Pilas, donde hay una presencia Kanu'l declarada abiertamente en los textos.
} 
más poderoso o bien, puede en que estas zonas siempre se mantuviese el culto a la serpiente acuática de una manera independiente.

Por último, la poca información de monumentos provenientes de Becán y Chactún no permite establecer una interpretación clara, pues las dinastías locales tuvieron un nuevo auge después de la caída de los Kanu'l durante el siglo viII (Šprajc, 2015: 95), razón que los pudo llevar a adoptar practicas rituales que involucraron el uso de ciertos símbolos de prestigio como los tocados de serpiente acuática. Después de la caída de los Kanu’l los tocados pudieron adquirir prestigio por su asociación con un poder hegemónico precedente, algo similar a lo que ocurre con los símbolos teotihuacanos en el Clásico Tardío. ${ }^{19}$ Para el caso de Copán se sabe que mantuvo una alianza con Tikal. No obstante, la relación diplomática de la familia gobernante de Copán frente a los Kanu’l es hipotética, por lo que no se puede afirmar que hubiese una enemistad entre ellos. También debe tomarse en cuenta que K'ahk' Yipaj Chan K'awiil era hijo de K'ahk' Joplaj Chan K'awiil, quien fue el gobernante de Copán que reestableció la dinastía después de un hiato en la escritura que duró 17 años, por lo que es complicado afirmar que la dinastía de Copán era aliada de Tikal para esas fechas. No obstante, tampoco debemos caer en el error de relacionarla con los Kanu'l; sino que más bien su orientación política es desconocida aún.

\section{Interpretación de resultados}

El patrón de representación de tocados de serpiente acuática durante el Clásico Tardío sugiere el siguiente modelo de difusión. Aparece durante el siglo vil en Calakmul y su red política de aliados como La Corona y El Perú. Todos los portadores ostentan el título más alto de la jerarquía política y son mujeres y hombres que gobernaron en sus respectivas ciudades. Hacia finales del siglo vil y a principios del viII, el tocado empieza a ser representado en la región del Usumacinta. Primero aparece en Palenque y Pomoná, cuyas relaciones con las tierras bajas centrales en esta época aún son hipotéticas, pero hay elementos como los lazos sanguíneos mencionados en inscripciones que hubo entre gobernantes locales y miembros de la dinastía Kanu'l; así como una ritualidad compartida, mencionada al inicio, que apoyan la idea de una presencia Kanu'l en la zona. Posteriormente, en la segunda mitad del siglo viII, los tocados de serpiente acuática fueron utilizados en Yaxchilán, Bonampak y Laxtunich por diversos estratos de la élite como los sajal y los gobernantes. Los textos jeroglíficos de los monumentos ubicados en estas tres ciudades confirman que Yax Chit Juun Witz' Naah Kan fue personificado por los portadores de tocados de variantes 1, 2 y 3 . Otro dato que refuerza la presencia de los Kanu'l en el Usumacinta, es el origen de la madre de Yaxuun B'ahlam IV. Sin embargo, ya se ha hablado del posible discurso retórico que se manejó en dichas fechas.

19 Daniel Salazar Lama, comunicación personal, 2019. 
En el área del Petexbatún y La Pasión prevalecen los tocados de variante 1 y, aunque es difícil hablar de una presencia de los Kanu'l en la zona por la pérdida gradual del poder que la dinastía experimentaba durante el siglo vill, se puede considerar que la tradición perduró y se convirtió en una adaptación local del culto a la serpiente acuática. Es decir, el tocado de serpiente acuática sobrevivió y se fue transformando con el paso del tiempo en cultos locales practicados en los siglos vill y Ix.

Los tocados de serpiente acuática durante el Clásico Tardío muestran una distribución que favorece la hipótesis de que están relacionados con la dinastía Kanu'l, así como el hecho de que no haya ejemplares representados en monumentos relacionados con los enemigos de ésta, como la dinastía de Tikal. A pesar de que es una hipótesis ambiciosa, hay elementos que la sostienen, tales como la relación entre Yax Chit Juun Witz' Naah Kan y la dinastía Kanu'l en la onomástica, la ritualidad compartida y el protocolo visual basado en la indumentaria de mujeres relacionadas con Kanu'l. Es importante recalcar que este estudio se centró solamente en el Clásico Tardío, pues es cuando más ejemplares se conocen y el modelo construido aquí se basa en dicha periodicidad. No obstante, se tendría que analizar el patrón desde el Preclásico para así conocer el significado político de este tipo de tocados y sus variantes, si todos corresponden a una tradición pan maya o desde un inicio está vinculado a un grupo político.

\section{Otro tocado de serpiente acuática}

Los tocados de serpiente acuática de variantes 1,2 y 3 han sido analizados en los anteriores párrafos. No obstante, hace algunos años se propuso la existencia de otro tipo de tocado de serpiente acuática que se compone de una cabeza de ofidio con un par de volutas conformadas por pequeñas cuentas sobre su cabeza, que han sido interpretadas como gotas de agua o nubes (Figura $6 \mathrm{~A}-\mathrm{C}$ ) (Zamora, s.f.: 66). Visualmente, este tocado se deriva de una cabeza de ofidio con una sola voluta, la cual estaba acompañada de un nenúfar sobre una concha de mar durante el Clásico Temprano. Ejemplos de esto se observan en un diente de felino grabado, antes mencionado, proveniente de una colección privada (Figura 1B) y en las Estelas 63 y 24 de Copán. Es por lo que en este trabajo ha sido nombrado como variante 4 , pues de igual forma representan a la serpiente acuática con algunas variaciones que veremos en la Tabla 3.

\begin{tabular}{|l|l|l|l|}
\hline \multicolumn{2}{|c|}{ Variante 4 } & \multicolumn{2}{c|}{ Fig. 1B } \\
\hline Cabeza de ofidio labio hacia abajo & U & Cabeza de ofidio labio hacia abajo & U \\
\hline Volutas & V & Volutas & V \\
\hline Cabeza de ofidio labio hacia arriba ${ }^{20}$ & W & Nenúfar & Y \\
\hline & & Caparazón & X \\
\hline
\end{tabular}

Tabla 3. Rasgos diagnósticos de los tocados de variante 4.

${ }^{20}$ En esta variante de tocados encontramos que el labio puede ser hacia arriba o hacia abajo. 
Estos tocados fueron utilizados exclusivamente por mandatarios durante los siglos VII a Ix. La mayoría de los ejemplares que se conocen provienen de Dos Pilas, pero también aparecen en Tamarindito, Aguateca, Naranjo y Calakmul (Figura 5B). Es importante mencionar que los portadores del tocado de variante 4 en Dos Pilas y Naranjo comparten lazos consanguíneos a raíz del matrimonio de la Señora 6 Cielo con el gobernante de Naranjo.

Los tocados de variante 4 no aparecen en relación con el teónimo Yax Chit Juun Witz' Naah Kan, así que hay que considerar la posibilidad de que represente a otra entidad sobrenatural. Se ha propuesto que las volutas diagnósticas representan agua o nubes, lo que podría ser una referencia al dios de la lluvia Chaahk o a Yopaat, cuyo signo nominal incluye este elemento (Looper, 2003: 4; Pallán, 2009: 25; Zamora, s.f.: 66). En la Estela 13 de Naranjo, el tocado se relaciona con una referencia textual a la personificación de los dioses remeros y un aspecto de Yopaat (Colas, 2004: 274). Este tocado es idéntico al de las Estelas 9 de Calakmul; 17, 5 y 11 de Dos Pilas; 12, 6 y 7 de Naranjo; 3 de Tamarindito y 7 de Aguateca, así como el del Panel 19 de Dos Pilas. Sin embargo, la Estela 15 de Dos Pilas nos muestra un tocado acompañado de una máscara facial en forma de yelmo compuesto de un mosaico de teselas (ko'haw) que incluye el conjunto del nenúfar atado típico del tocado de serpiente acuática en la variante 1. Lo mismo ocurre en la Estela 1 de Ixkún, donde se observa al personaje del lado izquierdo con un tocado que lleva una cabeza de ofidio acompañada del nenúfar atado con un pescado y el par de volutas en lo alto.

Es complicado afirmar que el tocado de variante 4 representa a una sola entidad sobrenatural, pues el rostro con rasgos zoomorfos que lo compone muestra variaciones iconográficas. Los tocados de las Estelas 1 de Dos Pilas, 6, 7 y 13 de Naranjo, ilustran a un ser con el labio superior hacia abajo; mientras que en las Estelas 5, 9 y 11 de Dos Pilas, 12 de Naranjo y 3 de Tamarindito el labio superior está levantado. El ofidio con labio superior hacia abajo podría tratarse de una personificación del dios Yopaat, pues en la Estela 13 de Naranjo se manifiesta esta acción en la escritura jeroglífica. Es posible que en el Panel 19 de Dos Pilas haya una correspondencia entre el tocado de doble voluta y un signo no descifrado (K1) con el mismo elemento icónico que representa parte del nombre del portador (Felix Kupprat, comunicación personal, 2018). No obstante, falta estudiar más aquellos mascarones cuyas características iconográficas muestran a un ofidio con labio superior levantado.

Si bien las variantes de los tocados de variante 4 no nos dejan tener una interpretación certera de la entidad representada, podemos ver que su distribución está sumamente relacionada con la dinastía gobernante de Dos Pilas, que pudo utilizarlos como símbolo entre sus confederados. Es decir, la estrategia manejada por los Kanu'l en el siglo vil para desarrollar una red de aliados a través del uso de elementos visuales en prácticas rituales fue adoptada por su coligado Dos Pilas y distribuida entre los sitios ubicados en el Petexbatún (Aguateca y Tamarindito) y Petén (Naranjo). No obstante, esta hipótesis necesita ser desarrollada a profun- 
didad por los diversos problemas que ya he señalado al momento de conocer su origen. Esta variante será analizada en futuras investigaciones, pues por motivos de espacio no es posible abordar todos los componentes que presenta.

\section{Consideraciones finales}

En este trabajo se consideraron cuatro tipos de tocados de serpiente acuática representados durante el Clásico Tardío (Tabla 4). Con base en el análisis de la distribución temporal y espacial de las variantes 1,2 y 3 se puede señalar que estuvieron relacionadas con la dinastía Kanu'l durante el siglo vII e inicios del vill, de tal forma que su red política de aliados utilizó estos atributos de poder con el propósito de generar identidad entre las élites de diversos linajes, mientras que las apariciones tardías señalan que los tocados fueron adaptaciones locales con fines políticos en una fase post-Kanu'l (segunda mitad del siglo vill y ix). Es una evolución que experimenta este símbolo que surge como elemento pan maya, y que posteriormente es utilizado por los Kanu'l y sus aliados como una estrategia de integración, al grado de convertirse en un culto post-Kanu'l en época tardías. Sin embargo, no debemos olvidar que hay sitios en los que es complicado definir su orientación política, como Palenque y Yaxchilán. Además, hay otro tocado que posiblemente sea de serpiente acuática que está relacionado con la dinastía gobernante de Dos Pilas, que parece adoptar una estrategia de integración similar a la de los Kanu'l a través del uso de elementos visuales.

Falta aún por profundizar sobre la distribución de los monumentos dentro de la ciudad, lo que contribuirá al desarrollo de hipótesis que indiquen hacia qué público eran dirigidos los mensajes plasmados en ellos. ${ }^{21}$ De igual manera hace falta analizar el uso familiar del teónimo Yax Chit Juun Witz' Naah Kan entre la familia real de Yaxchilán, pues todos sus miembros personifican a la mencionada divinidad.

En este estudio solamente se analizaron los tocados de serpiente acuática desde el Clásico Tardío, faltaría por realizar un estudio más detallado desde el Preclásico para conocer su evolución y a qué necesidades responden.

\footnotetext{
21 Por ejemplo, en el caso de las Estelas 33 y 34 de El Perú, fueron colocadas en la Plaza 1, frente a la Estructura M12-35 que posiblemente fue un espacio ritual al que la población podía acceder para apreciar las ceremonias (Acuña, 2014: 55-56); mientras que la Escalinata Jeroglífica 2 de Yaxchilán se ubica frente al Templo 33 en la Gran Acrópolis, una zona de difícil acceso para la población, por lo que pudo tratarse de un mensaje dirigido a un grupo de élite más específico. Un análisis más detallado será presentado en el futuro, ya que se necesita indagar más acerca de los espacios públicos y sus significados, objetivo que rebasa el presente trabajo.
} 


\begin{tabular}{|c|c|c|c|c|c|}
\hline \multicolumn{6}{|c|}{ Tocados de variante 1} \\
\hline Sitio & Monumento & Personaje & Género & Fecha & Referencias \\
\hline Calakmul & Estela 9* & Ho' Hu'n? Sipiil? & $\mathrm{F}$ & 672 d.C. & $\begin{array}{l}\text { Dibujo de Eric Von } \\
\text { Euw, archivo del } \\
\text { CMHI }^{* *}(1977)\end{array}$ \\
\hline El Perú & Estela 33 & K'ihnich Bahlam II & M & 692 d.C. & $\begin{array}{l}\text { Wanyerka, 1996: } \\
\text { 74, figura 2; Acuña, } \\
\text { 2014: 61-62 } \\
\end{array}$ \\
\hline Palenque & $\begin{array}{l}\text { Porta } \\
\text { incensario }\end{array}$ & Ix Aj Pay K'ab & $\mathrm{F}$ & 692 d.C. & $\begin{array}{l}\text { Ruz, 1953: 91, figura } \\
\text { 9; Bernal, 2011: } 160\end{array}$ \\
\hline Dos Pilas & Estela 15 & Kokaaj K'awiil & $M$ & 721 d.C. & $\begin{array}{l}\text { Houston, 1993: 91, } \\
\text { Vega, 2014: 205, } \\
\text { figura } 99\end{array}$ \\
\hline Chactun & Estela 18 & Desconocido & $\mathrm{NI}$ & 731 d.C. & $\begin{array}{l}\text { Esparza, 2015: } 72 \text {, } \\
\text { figura } 4.47 a\end{array}$ \\
\hline Machaquila & Estela 10 & Etz'nab? Chaahk & M & 731 d.C.? & $\begin{array}{l}\text { Lacadena, 2011: } \\
\text { 214, figura } 6\end{array}$ \\
\hline Copan & Estela N & $\begin{array}{l}\text { K'ahk' Yipyaj } \\
\text { Chan K'awiil }\end{array}$ & $M$ & 761 d.C. & \begin{tabular}{|l|} 
Baudez, 1994: 80- \\
87, figura 34-35 \\
\end{tabular} \\
\hline Yaxchilán & Dintel 6 & K'an Tok Wayib & $M$ & $752-768$ d.C. & $\begin{array}{l}\text { Graham, 1982, figura } \\
\text { 23; Mathews, 1997: } \\
30\end{array}$ \\
\hline Machaquila & Estela 18 & Chak Bahlam & $M$ & 776 d.C. & $\begin{array}{l}\text { Chocón y Laporte, } \\
\text { 2002: } 21\end{array}$ \\
\hline Yaxchilán & Esc. J. 2: 1 & $\begin{array}{l}\text { ¿Yaxuun Bahlam } \\
\text { III? }\end{array}$ & ¿M? & $752-768$ d.C. & $\begin{array}{l}\text { Graham, 1982, figura } \\
\text { 156; Mathews, 1997: } \\
35\end{array}$ \\
\hline Bonampak & Estela 2* & \begin{tabular}{|l|} 
Ix Yax Chit Juun \\
Witz' Naah Kan \\
\end{tabular} & $\mathrm{F}$ & 789 d.C. & $\begin{array}{l}\text { Mathews, 1978:62, } \\
\text { fig. } 2 \\
\end{array}$ \\
\hline Ixkun & Estela 1 & ¿? & $M$ & 790 d.C. & $\begin{array}{l}\text { Graham, 1980, figura } \\
\text { 137; Laporte et al., } \\
\text { 1994: } 42\end{array}$ \\
\hline Ixkun & Estela 4 & ¿? & $M$ & 790 d.C. & $\begin{array}{l}\text { Graham, 1980, figura } \\
\text { 148; Laporte et al., } \\
\text { 1994: } 45\end{array}$ \\
\hline La Amelia & Estela 1 & $\begin{array}{|ll|}\text { Lajchan } & \text { K'awiil } \\
\text { Ajaw Bot } & \\
\end{array}$ & $M$ & 807 d.C. & $\begin{array}{l}\text { Barrois y Tokovinine, } \\
\text { 2005: } 4 \text {, figura } 2 a\end{array}$ \\
\hline Machaquila & Estela 3 & $\begin{array}{l}\text { Siyaj K'in Chaahk } \\
\text { II }\end{array}$ & $M$ & 816 d.C. & $\begin{array}{l}\text { Graham, 1967, figura } \\
\text { 49; Lacadena, 2011: } \\
\text { 230, figura 10 }\end{array}$ \\
\hline Machaquila & Estela 4 & $\begin{array}{l}\text { Siyaj K'in Chaahk } \\
\text { II }\end{array}$ & $M$ & 820 d.C. & $\begin{array}{l}\text { Graham, 1967, figura } \\
\text { 51; Lacadena, 2011: } \\
\text { 230, figura 11 }\end{array}$ \\
\hline Machaquila & Estela 8 & Jun Tzak Tok' & M & 825 d.C. & $\begin{array}{l}\text { Lacadena, 2011: } \\
\text { 230, figura } 11\end{array}$ \\
\hline Machaquila & Estela 7 & Jun Tzak Tok' & $M$ & 830 d.C. & \begin{tabular}{|l} 
Graham, 1967, figura \\
57; Lacadena, 2011: \\
230, figura 12 \\
\end{tabular} \\
\hline Ceibal & Estela 11 & $\begin{array}{l}\text { Aj Balun Haabtal } \\
\text { Wat'ul K'atel }\end{array}$ & $M$ & 849 d.C. & $\begin{array}{l}\text { Just, 2007: 24, figura } \\
\text { 14; Graham, 1996: } \\
\text { figura } 34\end{array}$ \\
\hline
\end{tabular}




\begin{tabular}{|c|c|c|c|c|c|}
\hline $\begin{array}{ll}\text { Arroyo de } \\
\text { Piedra }\end{array}$ & Estela 2 & Chakbi[..]ahk & $M$ & 731 & $\begin{array}{l}\text { Houston y Mathews, } \\
\text { 1985: } 16 \text {, figura } 11 ; \\
\text { Escobedo et al., } \\
\text { 1995: } 398 \text {, figura } 3\end{array}$ \\
\hline Itzimite' & Estela 10 & Desconocido & $\mathrm{NI}$ & \begin{tabular}{|l|} 
Clásico \\
Tardío
\end{tabular} & Von Euw,1977: 25 \\
\hline \multicolumn{6}{|c|}{ Tocados de variante 2} \\
\hline Laxtunich & Dintel $2^{*}$ & Kokaaj Bahlam IV & $M$ & 769 d.C. & $\begin{array}{l}\text { Jackson, 2013: 133, } \\
\text { figura } 42\end{array}$ \\
\hline Palenque & Tablero Palacio & Janaab Pakal & $M$ & 720 d.C. & $\begin{array}{l}\text { Schele, 1979: 52, } \\
\text { figura 10; Bernal, } \\
2011: 321,325 \\
\end{array}$ \\
\hline Pomoná & Panel 1 & ¿? & $M$ & 771 d.C. & $\begin{array}{l}\text { Stuart, 2004: 3, } \\
\text { figura } 4\end{array}$ \\
\hline Pomoná & Panel 3 & ¿? & $M$ & Desconocido & $\begin{array}{l}\text { García Moll, 2005: } \\
\text { 113, 117, láminas } \\
\text { 6-17 }\end{array}$ \\
\hline Yaxchilán & Esc. J. 2: IV & $\begin{array}{l}\text { ¿Yaxuun Bahlam } \\
\text { IV? }\end{array}$ & $\mathrm{NI}$ & $752-768$ & $\begin{array}{l}\text { Graham, 1982, figura } \\
\text { 157; Mathews, 1997: } \\
35\end{array}$ \\
\hline Yaxchilán & Esc. J. 2: V & Desconocido & $\mathrm{NI}$ & $752-768$ & $\begin{array}{l}\text { Graham, 1982, figura } \\
\text { 158; Mathews, 1997: } \\
\text { 35 }\end{array}$ \\
\hline Yaxchilán & Esc. J. $2: \mathrm{X}$ & {$[\ldots] \mathrm{Jol}$} & $\mathrm{NI}$ & $752-768$ & $\begin{array}{l}\text { Graham, 1982, figura } \\
\text { 163; Mathews, 1997: } \\
35\end{array}$ \\
\hline Yaxchilán & Esc. J. 2: XII & K'an Tok Wayib & $M$ & $752-768$ & $\begin{array}{l}\text { Graham, 1982, figura } \\
\text { 164; Mathews, 1997: } \\
35\end{array}$ \\
\hline \multicolumn{6}{|c|}{ Tocados de variante 3} \\
\hline La Corona & $\begin{array}{|ll|}\text { Elemento } & 19 / \\
\text { Panel } 3 & \\
\end{array}$ & Ix Chak Tok Chahk & $\mathrm{F}$ & 662 d.C. & $\begin{array}{l}\text { Stuart et al., 2013: } \\
\text { 447; Stuart, Canuto y } \\
\text { Barrientos, 2015: } 9\end{array}$ \\
\hline Calakmul & \begin{tabular}{|l} 
Vasija \\
de \\
Schaffhausen
\end{tabular} & Ix $[\ldots] \operatorname{Tok}[\ldots]$ & $\mathrm{F}$ & 672 d.C. & $\begin{array}{l}\text { Prager, 2004: } 37, \\
\text { figura } 13\end{array}$ \\
\hline El Perú & Estela $34^{*}$ & Ix K'abel? & $\mathrm{F}$ & 692 d.C. & $\begin{array}{l}\text { Wanyerka, 1996: 73, } \\
\text { figura 1; Guenter, } \\
\text { 2014: 156-158 }\end{array}$ \\
\hline Palenque & Templo XIV & Ix Tz'akbu Ajaw & $\mathrm{F}$ & 685 d.C. & $\begin{array}{l}\text { Schele y Freidel, } \\
\text { 1990: 272, figura } \\
\text { VII.2; Bernal, 2011: } \\
\text { 323-324 }\end{array}$ \\
\hline La Corona & Panel 6 & Ho'? h'un? & $\mathrm{F}$ & 731 d.C. & $\begin{array}{l}\text { Martin, 2008: 1, } \\
\text { figura } 1\end{array}$ \\
\hline El Chorro & Estela 1 & Ix Sak Mo' Bahlam. & $\mathrm{F}$ & Desconocido & Mayer, 1989. \\
\hline Pomoná & Elemento 30 & Ix K'an Balun & $\mathrm{F}$ & Desconocido & $\begin{array}{l}\text { García Moll, 2005: } \\
\text { 113, 117, láminas } \\
6-17\end{array}$ \\
\hline Pomoná & $\begin{array}{|ll|}\text { Panel } & \text { de } \\
\text { Dallas } & \\
\end{array}$ & Ix K'an Balun & $\mathrm{F}$ & Desconocido & García Moll, 2005 \\
\hline
\end{tabular}




\begin{tabular}{|c|c|c|c|c|c|}
\hline Pomoy & Estela 3 & Ix Ook Ahiin & $\mathrm{F}$ & 700 d.C. & $\begin{array}{l}\text { Teufel, 2001: } 173 \text {, } \\
\text { figura } 260\end{array}$ \\
\hline Yaxchilán & Dintel 32 & Ix Uh Chan Lem? & $\mathrm{F}$ & ¿SIGLO VIII? & Mathews, 1997: 31 \\
\hline \multicolumn{6}{|c|}{ Tocados de variante 4} \\
\hline Calakmul & Estela 9 & Yihch'aak K'ahk' & $M$ & 672 d.C. & $\begin{array}{l}\text { Dibujo de Eric Von } \\
\text { Euw archivo del } \\
\text { CMHI }^{* *}(1977)\end{array}$ \\
\hline Dos Pilas & Estela 17 & Bajlaj Chan K'awiil & $M$ & 682 d.C. & $\begin{array}{l}\text { Vega, 2014: } 159, \\
\text { figura } 69\end{array}$ \\
\hline Dos Pilas & Estela 1 & Kokaaj K’awiil & $M$ & 706 d.C. & $\begin{array}{l}\text { Vega, 2014: 199, } \\
\text { figura } 93\end{array}$ \\
\hline Dos Pilas & Estela 5 & [...] Ti' K'awiil & $M$ & 716 d.C. & $\begin{array}{l}\text { Houston, 1993: } 82 \text {; } \\
\text { Vega, 2014: } 225 \text {, } \\
\text { figura } 112\end{array}$ \\
\hline Dos Pilas & Panel 19 & Kokaaj K’awiil & $M$ & 726 d.C. & $\begin{array}{l}\text { Houston 1993: 115; } \\
\text { Vega, 2014: } 234, \\
\text { figura 122 }\end{array}$ \\
\hline Dos Pilas & Estela 11 & Kokaaj K'awiil & $M$ & 731 d.C. & $\begin{array}{l}\text { Houston 1993: 93; } \\
\text { Vega, 2014: 204, } \\
\text { figura } 97\end{array}$ \\
\hline Naranjo & Estela 12 & $\begin{array}{l}\text { Itzam Kokaaj } \\
\text { K'awiil }\end{array}$ & $M$ & 800 d.C. & $\begin{array}{l}\text { Graham y Von Euw, } \\
\text { 1975: 35; Martin y } \\
\text { Grube, 2008: 82-83 }\end{array}$ \\
\hline Tamarindito & Estela $3^{22}$ & Desconocido & $M$ & Siglo VIII & $\begin{array}{l}\text { Gronemeyer, 2013: } \\
42\end{array}$ \\
\hline Naranjo & Estela 13 & $\begin{array}{l}\text { K'ahk' Ukalaw } \\
\text { Chan Chaahk }\end{array}$ & $M$ & 780 d.C. & $\begin{array}{l}\text { Graham y Von Euw, } \\
\text { 1975: 37; Martin y } \\
\text { Grube, 2008: 80-81 }\end{array}$ \\
\hline Naranjo & Estela 6 & $\begin{array}{l}\text { K'ahk' Ukalaw } \\
\text { Chan Chaahk }\end{array}$ & $M$ & 780 d.C. & $\begin{array}{l}\text { Graham y Von Euw, } \\
\text { 1975: 23; Martin y } \\
\text { Grube, 2008: 80-81 }\end{array}$ \\
\hline Aguateca & Estela 7 & Tahnte' K'innich & $M$ & 790 d.C. & $\begin{array}{l}\text { Graham, 1967; Vega, } \\
\text { 2014: 292, figura } 148\end{array}$ \\
\hline Naranjo & Estela 7 & $\begin{array}{l}\text { Itzam Kokaaj } \\
\text { K'awiil }\end{array}$ & $\mathrm{M}$ & 810 d.C. & $\begin{array}{l}\text { Martin y Grube, } \\
\text { 2008: } 82-83\end{array}$ \\
\hline
\end{tabular}

Tabla 4: Distribución espacial y temporal de los tocados de serpiente acuática.

* Monumentos donde se menciona que el portador del tocado de serpiente acuática personifica a Yax Chit Juun Witz' Naah Kan.

*** Corpus of Maya Hieroglyphic Inscriptions.

F: femenino/mujer, M: masculino/hombre, NI: no identificado, Esc. J.: Escalinata jeroglífica.

${ }^{22}$ En caso de la Estela 3 de Tamarindito se desconoce la fecha. Sin embargo, el análisis iconográfico de la misma ha dado pie a proponer que su elaboración pudo ser paralela a la de la Estela 11 de Dos Pilas, a mediados del siglo viII (Gronemeyer, 2013: 38). 


\section{Agradecimientos}

Agradezco al Posgrado en Estudios Mesoamericanos de la Universidad Nacional Autónoma de México por permitirme cursar mi maestría dentro de su programa, así como al Consejo Nacional de Ciencia y Tecnología (CONACYT) por haberme otorgado una beca a lo largo de mis estudios que culminan con la elaboración de este artículo. Infinitas gracias a Verónica Amellali Vázquez López y Felix Alexander Kupprat por dirigir este proyecto con sus valiosos comentarios y aportes desde su inicio. También agradezco a Luis Alberto Castro Gil por el apoyo con los programas de dibujo, así como a Daniel Salazar Lama, Alejandro Sheseña Hernández, Octavio Esparza Olguín y Roberto Romero Sandoval por sus puntuales comentarios y correcciones para la edición definitiva de este trabajo. Sin embargo, todo error o mala interpretación es responsabilidad mía. Por último, agradezco a mis padres y hermanos por el apoyo dado, así como a mi esposa Wendy, por siempre impulsarme y por su constante inspiración.

\section{Bibliografía}

Acuña, Mary Jane

2014 "Royal Alliance, Ritual Behavior and the Abandonment of the Royal Couple Building at El Perú-Waka'”, Archaeology at El Perú-Waka'. Ancient Maya Performances of Ritual, Memory, and Power, pp. 53-65, Olivia C. Navarro-Farr y Michelle Rich (eds.). Tucson: Universidad de Arizona.

Baron, Joanne P.

2016 Patron Gods and Patron Lords. The Semiotics of Classic Maya Community Cults. Boulder: University Press Colorado.

Barrois, Ramzy R. y Alexandre Tokovinine

2005 "El inframundo y el mundo celestial en el juego de pelota maya", XVIII Simposio de Investigaciones Arqueológicas en Guatemala, 2004. Guatemala: Museo Nacional de Arqueología y Etnología, <http://www.famsi.org/ reports/03101es/03barrois_tokovinine/03barrois_tokovinine.pdf $>$ [consultado el 17 abril de 2016].

Baudez, Claude-François

1994 Maya Sculpture of Copán. The Iconography. Norman y Londres: University of Oklahoma Press.

Bernal Romero, Guillermo

2011 "El señorío de Palenque durante la era de K'inich Janaahb' Pakal y K'inich Kan B'ahlam (615-702 d.C.)", tesis de doctorado en Estudios Mesoamericanos. México: Universidad Nacional Autónoma de México, Facultad de Filosofía y Letras, Instituto de Investigaciones Filológicas, Posgrado en Estudios Mesoamericanos. 
Boot, Erik

2008 "At the Court of Itzam Nah Yax Kokaj Mut. Preliminary Iconography and Epigraphic Analysis of a Late Classic Vessel", Maya Vase Database, pp. 1-49, <http://www.mayavase.com/God-D-Court-Vessel.pdf> [consultado el 2 de diciembre de 2016].

Canuto, Marcelo A. y Tomas Barrientos Quezada

2010 "La Corona: un acercamiento a las políticas del reino Kaan desde un centro secundario del noroeste del Petén”, Estudios de Cultura Maya, XXXVII: 11-43. DOI: http://dx.doi.org/10.19130/iifl.ecm.2011.37.12.

2013 "The Importance of La Corona”, Mesoweb, pp. 1-5, <www.mesoweb.com/ LaCorona/LaCoronaNotes01.pdf> [consultado el 15 agosto de 2017].

Carrasco Vargas, Ramón

2011 "Yuknoom Yich'aak K'ahk' (Garra de Jaguar) (¿649-695? d.C.). Calakmul, Campeche", Arqueología Mexicana, XIX (110): 62-67.

Carrasco Vargas, Ramón, Sylviane Boucher, Paula Álvarez González, Vera Tiesler Blos, Valeria García Vierna, Renata García Moreno y Javier Vázquez Negrete

1999 "A Dynastic Tomb from Campeche, Mexico: New Evidence on Jaguar Paw, a Ruler of Calakmul”, Latin American Antiquity, 10 (1): 47-58. DOI: https://doi. org/10.2307/972210.

Chocón, Jorge E. y Juan Pedro Laporte

2002 "La ciudad de Machaquilá en el municipio de Poptún”, Reporte 16, Atlas Arqueológico de Guatemala, pp. 1-3. Guatemala: Instituto de Antropología e Historia de Guatemala.

Colas, Pierre Robert

2004 Sinn und Bedeutung klassischer Maya-Personennamen: Typologische Analyse von Anthroponymphrasen in den Hieroglypheninschriften der klassischen Maya-Kultur als Beitrag zur allgemeinen Onomastik. Markt Schwaben: Anton Saurwein (Acta Mesoamericana, 15).

Cordeiro Baqueiro, María

2008 "El tocado de Yuknoom Yich'ak K'ak, k'uhul ahaw del reino de Kaan”, Arqueología Mexicana, XVI (94): 78-83.

Demarest, Arthur A., Juan Antonio Valdez, Héctor L. Escobedo y Stephen D. Houston

1992 "Una tumba real en el centro ceremonial de Dos Pilas, Petén: excavaciones e implicaciones", V Simposio de Investigaciones Arqueológicas en Guatemala, 1991, pp. 283-296, Juan Pedro Laporte, Héctor Escobedo y S. Brady (eds.). Guatemala: Museo Nacional de Arqueología y Etnología.

Durkheim, Émile

1964 The Elementary Forms of the Religious Life. Londres: George Allen and Unwin. 
Escobedo, Héctor L., José S. Suasnávar, Heidy Quezada y Mónica Urquizú

1995 "Resultados de la tercera temporada de Arroyo de Piedra: la política maya desde la perspectiva de un centro secundario", VIII Simposio de Investigaciones Arqueológicas Guatemala, 1994, pp. 397-414, Juan Pedro Laporte y Héctor Escobedo (eds.). Guatemala: Museo Nacional de Arqueología y Etnología.

Esparza Olguín, Octavio

2015 "Documentación de los monumentos esculpidos”, Exploraciones arqueológicas en Chactún, Campeche, México, pp. 41-84, Ivan Šprajc (ed.). Ljubljana: Založba ZRC.

García Barrios, Ana

2014 "Difusión de dioses como modelo de control político: el caso del reino de Kanu'l y el Dios Chaahk", Socio-Political Strategies among the Maya from the Classic Period to the Present, pp. 51-66, Verónica Vázquez, Rogelio Valencia y Eugenia Gutiérrez (eds.), Oxford: Bar International Series.

García Barrios, Ana y Rogelio Valencia Rivera

2007 "El uso político del baile en el Clásico maya: el baile del K'awiil”, Revista Española de Antropología Americana, 37 (2): 23-38.

García Barrios, Ana y Verónica A. Vázquez López

2011 "The Weaving of Power: Women's Clothing and Protocol in the Seventh-Century Kingdom of Kaanu'l", Latin American Indian Literatures Journal. A Review of American Indian Texts and Studies, 27 (1): 59-95.

García Capistrán, Hugo

2012 "El reino de la serpiente bajo la mirada de sus aliados", Contributions in New World Archaeology. Maya Political Relations and Strategies, 4: 41-57.

2014 "Religión y política en el Clásico maya: dioses patronos como vía de legitimación de poder", Socio-Political Strategies among the Maya from the Classic Period to the Present, pp. 67-81, Verónica Vázquez, Rogelio Valencia y Eugenia Gutiérrez (eds.). Oxford: Bar International Series, 51-66.

García Moll, Roberto

2005 Pomoná: un sitio del Clásico maya en las colinas tabasqueñas. México: Instituto Nacional de Antropología e Historia.

Gómez Palacios, Ricardo

2015 "El tocado como símbolo de poder. Un análisis iconográfico de algunos tocados de Yaxuun B'ahlam IV", tesis de licenciatura en Historia. México: Universidad Nacional Autónoma de México, Facultad de Filosofía y Letras.

Graham, Ian

1967 Archaeological Explorations in El Peten, Guatemala. New Orleans: Tulane University (Middle American Research Institute, 33).

1980 Corpus of Maya Hieroglyphic Inscriptions, vol. 2, part 3: Ucanal, Ixkun, Naranjo. 
Cambridge: Peabody Museum of Archaeology and Ethnography, Harvard University.

1982 Corpus of Maya Hieroglyphic Inscriptions, vol. 3, part 3: Yaxchilán. Cambridge: Peabody Museum of Archaeology and Ethnography, Harvard University.

1996 Corpus of Maya Hieroglyphic Inscriptions, vol. 7, part 1: Seibal. Cambridge: Peabody Museum of Archaeology and Ethnography, Harvard University.

Graham, Ian y Eric von Euw

1975 Corpus of Maya Hieroglyphic Inscriptions, vol. 2, part 1: Naranjo. Cambridge: Peabody Museum of Archaeology and Ethnography, Harvard University.

Gronemeyer, Sven

2013 The Monuments and Inscriptions of Tamarindito, Peten, Guatemala. Markt Schwabe: Verlag Anton Saurwein (Acta Mesoamericana, 25).

Grube, Nikolai

1995 "Palenque in the Maya World”, Octava Mesa Redonda de Palenque, pp. 12-13, Martha J. Macri y Jan McHargue (eds.). San Francisco: Pre-Columbian Art Institute.

Grube, Nikolai

2001 "Los nombres de los gobernantes mayas", Arqueología Mexicana, IX (50): $72-77$.

Guenter, Stanley P.

2014 “The Epigraphy of El Perú-Waka’”, Archaeology at El Perú-Waka'. Ancient Maya Performances of Ritual, Memory, and Power, pp. 147-166, Olivia C. Navarro-Farr y Michelle Rich (eds.). Tucson: Universidad de Arizona.

Helmke, Christophe, Christopher R. Andres, Shawn G. Morton y Gabriel D. Wrobel

2015 "For Love of the Game: The Ballplayer Panels of Tipan Chen Uitz in Light of Late Classic Athletic Hegemony”, The Pari Journal, 16 (2): 1-30.

Helmke, Christophe y Jesper Nielsen

2015 "The Defeat of the Great Bird in Myth and Royal Pageantry: A Mesoamerican Myth in a Comparative Perspective", Comparative Mythology, 1 (1): 23-60.

Helmke, Christophe y Jaime Awe

2016 "Sharper than a Serpent's Tooth: A Tale of the Snake-Head Dynasty as Recounted on Xunantunich Panel 4", The Pari Journal, 17 (2): 1-22.

Helmke, Christophe y Felix Kupprat

2016 "Where Snakes Abound: Supernatural Places of Origin and Founding Myths in the Titles of Classic Maya Kings", Places of Power and Memory in Mesoamerica's Past and Present: How Sites, Toponyms and Landscapes Shape History and Remembrance, pp. 33-83, Daniel Graña-Behrens (ed.). Berlin: Ibero-Amerikanisches Institut, Gebr Mann (Estudios Indiana, 9). 
Houston, Stephen

1993 Hieroglyphics and History at Dos Pilas. Dynastic Politics of the Classic Maya. Austin: University of Texas Press.

2017 “On Dragons, Whales and Wits'”, Maya Decipherment: Ideas of Ancient Maya Writing and Iconography, <https://decipherment.wordpress.com/2017/03/20/ on-dragons-whales-and-wits/> [consultado el 21 marzo de 2017].

Houston, Stephen y Peter Mathews

1985 The Dynastic Sequence of Dos Pilas, Guatemala. San Francisco: Pre-ColumbianArt Research Institute.

Houston, Stephen y David Stuart

1998 "The Ancient Maya Self: Personhood and Portraiture in the Classic Period”, Anthropology and Aesthetics, 33: 73-101. DOI: https://doi.org/10.1086/ RESv33n1ms20167002.

Ishihara, Reiko, Karl Taube y Jaime J. Awe

2006 "The Water Lily Serpent Stucco Masks at Caracol, Belize", Archaeological Investigations in the Eastern Maya Lowlands: Papers of the 2005 Belize Archaeology Symposium, pp. 211-222, John Morris, Sherilyne Jones, Jaime Awe y Christophe Helmke (eds.). Belmopan: Institute of Archaeology, National Institute of Culture and History.

Jackson, Sarah E.

2013 Politics of the Maya Court. Hierarchy and Change in the Late Classic Period. Norman: University of Oklahoma Press.

Just, Bryan R.

2007 "Las estelas del siglo Ix en Machaquilá y Ceibal”, FAMSI, <http://www.famsi.org/reports/01050es/01050esJust01.pdf> [consultado el 29 de marzo de 2016].

Kerr, Justin

2008 Maya Vase Database: An Archive of Rollout Photographs, <http://research.mayavase.com/kerrmaya_list.php?_allSearch $=\&$ hold_search $=\& \mathrm{x}=0 \& \mathrm{y}=0 \&$ vase number $=1162 \&$ date_added $=\&$ ms_number $=\& \overline{s i t e}=>$ [consultado el $23 \mathrm{de}$ junio de 2015].

Kupprat, Felix Alexander

2015 "La memoria cultural y la identidad maya en el periodo Clásico: una propuesta de método y su aplicación a los casos de Copán y Palenque en el siglo vil d.C.", tesis de doctorado en Estudios Mesoamericanos. México: Universidad Nacional Autónoma de México, Facultad de Filosofía y Letras, Instituto de Investigaciones Filológicas, Posgrado en Estudios Mesoamericanos.

Lacadena García-Gallo, Alfonso

2011 "Historia y ritual dinásticos en Machaquilá (Petén, Guatemala)”, Revista Espa- 
ñola de Antropología Americana, 41 (1): 205-240. DOI: https://doi.org/10.5209/ rev_REAA.2011.v41.n1.9.

Laporte, Juan Pedro, Héctor Escobedo, Paulino I. Morales, Julio A. Roldan, Rolando Torres, Oswaldo Gómez y Yolanda Fernández

1994 "Ixkún. Entidad política del noroeste de las Montañas Mayas”, Mayab, 9: $31-48$

Le Fort, Geneviéve

2002 "El traje real entre los mayas de la época clásica (250-900 d.C.)", Actas de las II Jornadas internacionales sobre textiles precolombinos, pp. 27-44, Victoria Solanilla (ed.). Barcelona: Universidad Autónoma de Barcelona.

Looper, Matthew G.

2003 Lightning Warrior: Maya Art and Kingship at Quirigua. Austin: University of Texas Press.

2004 “A 'Macaw Face Headband' Dance on Site R Lintel 5”, Glyph Dwellers, 18: 1-5, $<$ https://nas.ucdavis.edu/sites/nas.ucdavis.edu/files/attachments/R18.pdf > [consultado el 11 de marzo de 2017].

López Austin, Alfredo

1973 Hombre-Dios, religión y política en el mundo náhuatl. México: Universidad Nacional Autónoma de México, Instituto de Investigaciones Históricas.

Martin, Simon

2008 "Wives and Daughters on the Dallas Altar", Mesoweb, <http://www.mesoweb. com/articles/martin/Wives\&Daughters.pdf $>$ [consultado el 3 de marzo de 2017].

Martin, Simon y Nikolai Grube

1994 "Evidence for Macro-Political Organization Amongst Classic Maya Lowland States", Mesoweb, pp. 1-62, < http://www.mesoweb.com/articles/martin/Macro-Politics.pdf $>$ [consultado el 14 de mayo de 2018].

2008 Chronicle of the Maya Kings and Queens. Londres: Thames \& Hudson.

Mathews, Peter

1978 "Notes on the Dynastic Sequence of Bonampak, Part 1", Third Palenque Round Table, Part 2, pp. 60-73, Merle Greene Robertson (coord.). Austin: University of Texas Press.

1997 La escultura de Yaxchilán. México: Instituto Nacional de Antropología e Historia.

Mayer, Karl Herbert

1989 Maya Monuments: Sculptures from Unknown Provenance, Supplement 2, Berlín: Verlag von Flemming.

Morley, Sylvanus G.

1920 The Inscriptions at Copan. Washington: Carnegie Institution of Washington. 
Morselli Barbieri, Simonetta

2004 "El tocado de los gobernantes en las representaciones escultóricas de Tikal: propuesta para una lectura iconográfica", tesis de maestría en Estudios Mesoamericanos. México: Universidad Nacional Autónoma de México, Facultad de Filosofía y Letras, Instituto de Investigaciones Filológicas, Posgrado en Estudios Mesoamericanos.

Nehammer Knub, Julie, Simone Thun y Christophe Helmke

2009 "The Divine Rite of Kings: An Analysis of Classic Maya Impersonation Statements", The Maya and their Sacred Narratives: Text and Context in Maya Mythologies, pp. 177-195, Geneviève Le Fort, Raphaël Gardiol, Sebastian Matteo y Christophe Helmke (eds.). Markt Schwaben: Verlag Anton Saurwein (Acta Mesoamericana, 20).

Pallán Gayol, Carlos

2009 "The Many Faces of Chaahk: Exploring the Role of a Complex and Fluid Entity within Myth, Religion and Politics", The Maya and their Sacred Narratives: Text and Context in Maya Mythologies, pp.17-40, Geneviève Le Fort, Raphaël Gardiol, Sebastian Matteo y Christophe Helmke (eds.). Markt Schwaben: Verlag Anton Saurwein (Acta Mesoamericana, 20).

Parmington Alexander

2003 "Classic Maya Status and the Subsidiary 'Office' of Sajal. A comparative Study of Status as Represented in Costume and Composition in the Iconography of Monuments", Mexicon, 25 (2): 46-53.

Paulinyi, Zoltán

2001 "Los señores con tocado de borlas. Un estudio sobre el Estado teotihuacano", Ancient Mesoamerica, 12: 1-30.

Prager, Christian M.

2004 "A Classic Maya Ceramic Vessel from the Calakmul Region in the Museum zu Allerheiligen, Schaffhausen, Switzerland”, Human Mosaic, 35 (1): 31-40.

2013 "Übernatuirliche Akteure in der Klassischen Maya-Religion: Eine Untersuchung zi intrakultureller Variation und Stabilität am Beispiel des k'uh 'Götter'-Konzepts in den religiösen Vorstellungen and Überzeugungen Klassischer Maya-Eliten (250-900 c.Chr.)”, tesis de doctorado en Antropología de las Américas. Bonn: Universität Bonn, Philosophische Fakultät.

Ramírez de la Fuente, Beatriz y Alfonso Arellano (editores)

2001 El hombre maya en la plástica antigua. México: Universidad Nacional Autónoma de México, Coordinación de Humanidades.

Reese-Taylor, Kathryn, Peter Mathews, Julia Guernsey y Marlene Fritzler

2009 "Warrior Queens among the Classic Maya", Blood and Beauty: Organized Violence in the Art and Archaeology of Mesoamerica and Central America, pp. 39-72, Heather Orr y Rex Koontz (eds.). Los Angeles: University of California. 
Robertson, Merle Greene

1974 "Quadripartite Badge; A Badge of Rulership”, Primera Mesa Redonda de Palenque, pp. 77-93, Merle Greene Robertson y Louis Stevenson School (eds.). California: Pre-Columbian Art Research.

Ruz Lhuillier, Alberto

1953 "Exploraciones arqueológicas en Palenque: 1953", Anales del Instituto Nacional de Antropología e Historia, X (39): 69-116.

Schele, Linda

1979 "Genealogical Documentation on the Tri-Figure Panels at Palenque”, Tercera Mesa Redonda de Palenque, pp. 41-70, Merle Greene Robertson y Donnan Call Jeffers (eds.). San Francisco: Pre-Columbian Art Institute.

Schele, Linda y David A. Freidel

1990 A Forest of Kings: The Untold Story of the Ancient Maya. New York: William Morrow and Company.

Schele, Linda y Mary E. Miller

1986 The Blood of Kings. Dynasty and Ritual in Maya Art. New York: Kimbell Art Museum.

Šprajc, Ivan

2015 Exploraciones arqueológicas en Chactún, Campeche, México. Ljubljana: Založba ZRC.

Stone, Andrea

1986 "Aspects of Impersonation in Classic Maya Art", Sexta Mesa Redonda de Palenque, pp. 194-202, Merle Greene Robertson y Virginia Fields (eds.). Norman, University of Oklahoma Press.

Stuart, David

2004 "New Year Records in Classic Maya Inscriptions”, The Pari Journal, 5 (2): 1-6, <http://www.mesoweb.com/pari/publications/journal/502/NewYear.html> [consultado el 18 de abril de 2017]

2005 The Inscriptions from Temple XIX at Palenque. A Commentary. San Francisco: The Pre-Columbian Art Research Institute.

2007 "Reading the Water Serpent as WITZ”, Maya Decipherment: Ideas of Ancient Maya Writing and Iconography, <https://decipherment.wordpress.com/ 2007/04/13/reading-the-water-serpent/> [consultado el 16 de agosto de 2016].

Stuart, David, Nikolai Grube, Linda Schele y Floyd Lounsbury

1989 “Stela 63, A New Monument from Copan”, Copan Notes, 56: 296-387.

Stuart, David, Peter Mathews, Marcello Canuto, Tomás Barrientos, Stanley Guenter y Joanne Baron

2013 “Un esquema de la historia y epigrafía de La Corona”, XXVII Simposio de Inves- 
tigaciones Arqueológicsa en Guatemala, pp. 435-447, Bárbara Arroyo, Luis Méndez y Andrea Rojas (eds.). Guatemala: Instituto de Antropología e Historia.

Stuart, David, Marcelo Canuto y Tomás Barrientos

2015 "The Nomenclature of La Corona Sculpture", La Corona Notes, 1 (2): 1-9, <http://www.mesoweb.com/LaCorona/LaCoronaNotes02.html> [consultado el 29 de junio de 2016].

Taylor, Dicey

1984 "Classic Maya Costume: Regional Types of Dress", tesis de doctorado en Antropología. Yale: Yale University.

Teufel, Stefanie

2001 "Matrimonios diplomáticos: mujeres en la corte", Los mayas, una civilización milenaria, pp. 172-173, Nikolai Grube (ed.). Colonia: Könemann.

Tremain, Cara Grace

2017 "A Study of Dress and Identity in the Late Classic Maya Court", tesis de doctorado en Arqueología. Calgary: University of Calgary, Graduated Program in Archaeology.

Vázquez López, Verónica Amellali

2015 "Dinastías, linajes y casas: las unidades sociales mayas en el ámbito político en los Kanu’l en el Clásico Tardío", tesis de doctorado en Estudios Mesoamericanos. México: Universidad Nacional Autónoma de México, Facultad de Filosofía y Letras, Instituto de Investigaciones Filológicas, Posgrado de Estudios Mesoamericanos.

2016 "Pactos y matrimonios como estrategias sociopolíticas entre los Kanu’l y sus aliados en el Clásico Tardío”, ponencia presentada en el X Congreso Internacional de Mayistas, 26 de junio a 2 de julio de 2016, Izamal, Yucatán, México.

2017 "Pact and Marriage: Sociopolitical Strategies of the Kanu'l Dynasty and Its Allies During the Late Classic Period", Contributions in New World Archaeology, 11: $9-48$.

Vázquez López, Verónica Amellali y Felix Kupprat

2018 "Pertenencia y transmisión de nombres entre los Kanu’l en el Clásico Tardío”, Estudios de Cultura Maya, LI: 75-110. DOI: https://doi.org/10.19130/iifl. ecm.2018.51.851.

Vega Villalobos, María Elena

2014 "La entidad política de Dos Pilas: un señorío maya del periodo Clásico", tesis de doctorado en Estudios Mesoamericanos. México: Universidad Nacional Autónoma de México, Facultad de Filosofía y Letras, Instituto de Investigaciones Filológicas, Posgrado de Estudios Mesoamericanos.

Velásquez García, Erik

2006 "Iconografía real de K’ahk' Tiliw Chan Yo’aat: política y fundación del mundo en Quiriguá, Guatemala”, La imagen política. XXV Coloquio Internacional de His- 
toria del Arte: pp. 113-146, Cuauhtémoc Medina (ed.). México: Universidad Nacional Autónoma de México, Instituto de Investigaciones Estéticas.

2010 "Naturaleza y papel de las personificaciones en los rituales mayas, según las fuentes epigráficas, etnohistóricas y lexicográficas”, El ritual en el mundo maya. De lo privado a lo público, pp. 203-234, Andrés Ciudad Ruiz, María Josefa Iglesias y Miguel Sorroche (eds.). Madrid: Sociedad Española de Estudios Mayas.

Von Euw, Eric

1977 Corpus of Maya Hieroglyphic Inscriptions, vol. 4, part 1: Iztminte, PixTzum. Cambridge: Peabody Museum of Archaeology and Ethnography, Harvard University.

Wanyerka, Phil

1996 "A Fresh Look at a Maya Masterpiece", Cleveland Studies in the History of Art, 1: 72-97.

Zamora Moya, María Auxiliadora

s.f. La serpiente de agua en el periodo Clásico maya, manuscrito en la Universidad Complutense de Madrid.

Ricardo Gómez Palacios. Mexicano. Licenciado en Historia por la Facultad de Filosofía y Letras de la Universidad Nacional Autónoma de México, cursa la maestría en el programa de Posgrado en Estudios Mesoamericanos de la misma universidad y se especializa en la iconografía maya. Su proyecto de investigación actual se titula "Los tocados mayas en el discurso político. Un estudio de caso de los tocados de serpiente acuática durante el Clásico Tardío”. Es autor en la Guía de Historia de la educación en México, Guía para los alumnos de pedagogía en la modalidad de universidad abierta, y colaborador en el libro de texto para bachillerato, Historia universal inédita.

rgomezpalacios28@gmail.com 